TUM-HEP-523/03

hep-ph/0307203

July 2003

\title{
CP Violation in B and K Decays: 2003
}

\author{
Andrzej J. Buras \\ Technische Universität München \\ Physik Department \\ D-85748 Garching, Germany
}

\begin{abstract}
These lectures give a brief description of $\mathrm{CP}$ violation in $\mathrm{B}$ and $\mathrm{K}$ meson decays with particular emphasize put on the determination of the CKM matrix. The following topics will be discussed: i) The CKM matrix, the unitarity triangle and general aspects of the theoretical framework, ii) Particle-antiparticle mixing and various types of $\mathrm{CP}$ violation, iii) Standard analysis of the unitarity triangle, iv) The ratio $\varepsilon^{\prime} / \varepsilon$, v) The most important strategies for the determination of the angles $\alpha, \beta$ and $\gamma$ from B decays, vi) Rare decays $K^{+} \rightarrow \pi^{+} \nu \bar{\nu}$ and $K_{L} \rightarrow \pi^{0} \nu \bar{\nu}$ and vii) Models with minimal flavour violation, in particular those with universal extra dimensions.
\end{abstract}

Lectures given at

41. Schladming School in Theoretical Physics

Schladming, February 22-28, 2003 



\section{CP Violation in B and K Decays: 2003}

Andrzej J. Buras

\section{Introduction}

\subsection{Preface}

$\mathrm{CP}$ violation in $\mathrm{B}$ and $\mathrm{K}$ meson decays is not surprisingly one of the central topics in particle physics. Indeed, CP-violating and rare decays of $\mathrm{K}$ and $\mathrm{B}$ mesons are very sensitive to the flavour structure of the Standard Model (SM) and its extensions. In this context a very important role is played by the Cabibbo-Kobayashi-Maskawa (CKM) matrix [1 2] that parametrizes the weak charged current interactions of quarks: its departure from the unit matrix is the origin of all flavour violating and $\mathrm{CP}$-violating transitions in the $\mathrm{SM}$ and its simplest extensions.

One of the important questions still to be answered, is whether the CKM matrix is capable to describe with its the four parameters all weak decays that include in addition to tree level decays mediated by $W^{ \pm}$-bosons, a vast number of one-loop induced flavour changing neutral current transitions involving gluons, photon, $W^{ \pm}, Z^{0}$ and $H^{0}$. The latter transitions are responsible for rare decays and $\mathrm{CP}$-violating decays in the SM. This important role of the CKM matrix is preserved in any extension of the SM even if more complicated extentions may contain new sources of flavour violation and $\mathrm{CP}$ violation.

The answer to this question is very challenging because the relevant rare and $\mathrm{CP}$-violating decays have small branching ratios and are often very difficult to measure. Moreover, as hadrons are bound states of quarks and antiquarks, the determination of the CKM parameters requires in many cases a quantitative control over QCD effects at long distances where the existing non-perturbative methods are not yet satisfactory.

In spite of these difficulties, we strongly believe that this important question will be answered in this decade. This belief is based on an impressive progress in the experimental measurements in this field and on a similar progress made by theorists in perturbative and to a lesser extend nonperturbative QCD calculations. The development of various strategies for the 
determination of the CKM parameters, that are essentially free from hadronic uncertainties, is also an important ingredient in this progress. A recent account of these joined efforts by experimentalist and theorists is given in [3].

These lecture notes provide a rather non-technical description of the decays that are best suited for the determination of the CKM matrix. We will also briefly discuss the ratio $\varepsilon^{\prime} / \varepsilon$ that from the present perspective is not suited for a precise determination of the CKM matrix but is interesting on its own. There is unavoidably an overlap with our Les Houches 4], Lake Louise [5], Erice [6] and Zacatecas [7] lectures and with the reviews [8] and 9]. On the other hand new developments until the summer 2003 have been taken into account, as far as the space allowed for it, and all numerical results have been updated. Moreover the discussion of the strategies for the determination of the angles $\alpha, \beta$ and $\gamma$ in the unitarity triangle goes far beyond our previous lectures.

We hope that these lecture notes will be helpful in following the new developments in this exciting field. In this respect the recent books [10, 11, 12, the working group reports $3,13,14,15$, and most recent reviews [16] are also strongly recommended.

\subsection{CKM Matrix and the Unitarity Triangle}

The unitary CKM matrix [1, 2] connects the weak eigenstates $\left(d^{\prime}, s^{\prime}, b^{\prime}\right)$ and the corresponding mass eigenstates $d, s, b$ :

$$
\left(\begin{array}{c}
d^{\prime} \\
s^{\prime} \\
b^{\prime}
\end{array}\right)=\left(\begin{array}{ccc}
V_{u d} & V_{u s} & V_{u b} \\
V_{c d} & V_{c s} & V_{c b} \\
V_{t d} & V_{t s} & V_{t b}
\end{array}\right)\left(\begin{array}{c}
d \\
s \\
b
\end{array}\right) \equiv \hat{V}_{\mathrm{CKM}}\left(\begin{array}{c}
d \\
s \\
b
\end{array}\right) .
$$

Many parametrizations of the CKM matrix have been proposed in the literature. The classification of different parametrizations can be found in [17. While the so called standard parametrization 18

$$
\hat{V}_{\mathrm{CKM}}=\left(\begin{array}{ccc}
c_{12} c_{13} & s_{12} c_{13} & s_{13} e^{-i \delta} \\
-s_{12} c_{23}-c_{12} s_{23} s_{13} e^{i \delta} & c_{12} c_{23}-s_{12} s_{23} s_{13} e^{i \delta} & s_{23} c_{13} \\
s_{12} s_{23}-c_{12} c_{23} s_{13} e^{i \delta} & -s_{23} c_{12}-s_{12} c_{23} s_{13} e^{i \delta} & c_{23} c_{13}
\end{array}\right),
$$

with $c_{i j}=\cos \theta_{i j}$ and $s_{i j}=\sin \theta_{i j}(i, j=1,2,3)$ and the complex phase $\delta$ necessary for $\mathrm{CP}$ violation, should be recommended [19] for any numerical analysis, a generalization of the Wolfenstein parametrization [20] as presented in 21] is more suitable for these lectures. On the one hand it is more transparent than the standard parametrization and on the other hand it satisfies the unitarity of the CKM matrix to higher accuracy than the original parametrization in [20].

To this end we make the following change of variables in the standard parametrization (2) 21, 22

$$
s_{12}=\lambda, \quad s_{23}=A \lambda^{2}, \quad s_{13} e^{-i \delta}=A \lambda^{3}(\varrho-i \eta)
$$


where

$$
\lambda, \quad A, \quad \varrho, \quad \eta
$$

are the Wolfenstein parameters with $\lambda \approx 0.22$ being an expansion parameter. We find then

$$
\begin{aligned}
& V_{u d}=1-\frac{1}{2} \lambda^{2}-\frac{1}{8} \lambda^{4}, \quad V_{c s}=1-\frac{1}{2} \lambda^{2}-\frac{1}{8} \lambda^{4}\left(1+4 A^{2}\right), \\
& V_{t b}=1-\frac{1}{2} A^{2} \lambda^{4}, \quad V_{c d}=-\lambda+\frac{1}{2} A^{2} \lambda^{5}[1-2(\varrho+i \eta)], \\
& V_{u s}=\lambda+\mathcal{O}\left(\lambda^{7}\right), \quad V_{u b}=A \lambda^{3}(\varrho-i \eta), \quad V_{c b}=A \lambda^{2}+\mathcal{O}\left(\lambda^{8}\right), \\
& V_{t s}=-A \lambda^{2}+\frac{1}{2} A \lambda^{4}[1-2(\varrho+i \eta)], \quad V_{t d}=A \lambda^{3}(1-\bar{\varrho}-i \bar{\eta})
\end{aligned}
$$

where terms $\mathcal{O}\left(\lambda^{6}\right)$ and higher order terms have been neglected. A nonvanishing $\eta$ is responsible for CP violation in the SM. It plays the role of $\delta$ in the standard parametrization. Finally, the bared variables in (8) are given by $21]$

$$
\bar{\varrho}=\varrho\left(1-\frac{\lambda^{2}}{2}\right), \quad \bar{\eta}=\eta\left(1-\frac{\lambda^{2}}{2}\right) .
$$

Now, the unitarity of the CKM-matrix implies various relations between its elements. In particular, we have

$$
V_{u d} V_{u b}^{*}+V_{c d} V_{c b}^{*}+V_{t d} V_{t b}^{*}=0 .
$$

The relation (10) can be represented as a "unitarity" triangle in the complex $(\bar{\varrho}, \bar{\eta})$ plane. One can construct additional five unitarity triangles [23] corresponding to other unitarity relations.

Noting that to an excellent accuracy $V_{c d} V_{c b}^{*}$ is real with $\left|V_{c d} V_{c b}^{*}\right|=A \lambda^{3}+$ $\mathcal{O}\left(\lambda^{7}\right)$ and rescaling all terms in (10) by $A \lambda^{3}$ we indeed find that the relation (10) can be represented as the triangle in the complex $(\bar{\varrho}, \bar{\eta})$ plane as shown in fig. 11 Let us collect useful formulae related to this triangle:

- We can express $\sin \left(2 \phi_{i}\right), \phi_{i}=\alpha, \beta, \gamma$, in terms of $(\bar{\varrho}, \bar{\eta})$. In particular:

$$
\sin (2 \beta)=\frac{2 \bar{\eta}(1-\bar{\varrho})}{(1-\bar{\varrho})^{2}+\bar{\eta}^{2}} .
$$

- The lengths $C A$ and $B A$ are given respectively by

$$
\begin{aligned}
& R_{b} \equiv \frac{\left|V_{u d} V_{u b}^{*}\right|}{\left|V_{c d} V_{c b}^{*}\right|}=\sqrt{\bar{\varrho}^{2}+\bar{\eta}^{2}}=\left(1-\frac{\lambda^{2}}{2}\right) \frac{1}{\lambda}\left|\frac{V_{u b}}{V_{c b}}\right| \\
& R_{t} \equiv \frac{\left|V_{t d} V_{t b}^{*}\right|}{\left|V_{c d} V_{c b}^{*}\right|}=\sqrt{(1-\bar{\varrho})^{2}+\bar{\eta}^{2}}=\frac{1}{\lambda}\left|\frac{V_{t d}}{V_{c b}}\right| .
\end{aligned}
$$




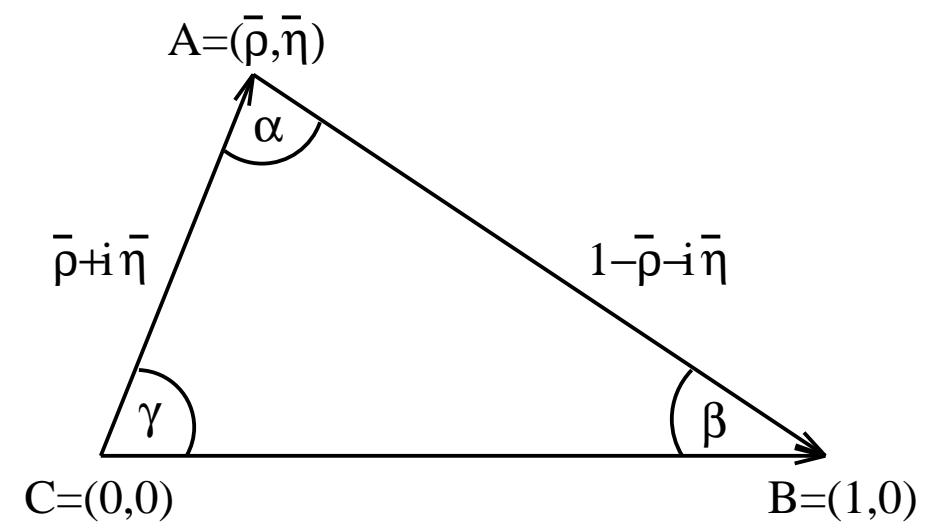

Fig. 1. Unitarity Triangle.

- The angles $\beta$ and $\gamma=\delta$ of the unitarity triangle are related directly to the complex phases of the CKM-elements $V_{t d}$ and $V_{u b}$, respectively, through

$$
V_{t d}=\left|V_{t d}\right| e^{-i \beta}, \quad V_{u b}=\left|V_{u b}\right| e^{-i \gamma} .
$$

- The unitarity relation (10) can be rewritten as

$$
R_{b} e^{i \gamma}+R_{t} e^{-i \beta}=1 .
$$

- The angle $\alpha$ can be obtained through the relation

$$
\alpha+\beta+\gamma=180^{\circ} \text {. }
$$

Formula (15) shows transparently that the knowledge of $\left(R_{t}, \beta\right)$ allows to determine $\left(R_{b}, \gamma\right)$ through

$$
R_{b}=\sqrt{1+R_{t}^{2}-2 R_{t} \cos \beta}, \quad \cot \gamma=\frac{1-R_{t} \cos \beta}{R_{t} \sin \beta} .
$$

Similarly, $\left(R_{t}, \beta\right)$ can be expressed through $\left(R_{b}, \gamma\right)$ :

$$
R_{t}=\sqrt{1+R_{b}^{2}-2 R_{b} \cos \gamma}, \quad \cot \beta=\frac{1-R_{b} \cos \gamma}{R_{b} \sin \gamma} .
$$

These relations are remarkable. They imply that the knowledge of the coupling $V_{t d}$ between $t$ and $d$ quarks allows to deduce the strength of the corresponding coupling $V_{u b}$ between $u$ and $b$ quarks and vice versa.

The triangle depicted in fig. 1] $\left|V_{u s}\right|$ and $\left|V_{c b}\right|$ give the full description of the CKM matrix. Looking at the expressions for $R_{b}$ and $R_{t}$, we observe that within the SM the measurements of four CP conserving decays sensitive to $\left|V_{u s}\right|,\left|V_{u b}\right|,\left|V_{c b}\right|$ and $\left|V_{t d}\right|$ can tell us whether CP violation $(\bar{\eta} \neq 0$ or $\gamma \neq 0, \pi)$ is predicted in the SM. This fact is often used to determine the angles of the unitarity triangle without the study of CP-violating quantities. 


\subsection{The Special Role of $\left|V_{u s}\right|,\left|V_{u b}\right|$ and $\left|V_{c b}\right|$}

What do we know about the CKM matrix and the unitarity triangle on the basis of tree level decays? Here the semi-leptonic K and B decays play the decisive role. The present situation can be summarized by [3]

$$
\begin{aligned}
& \left|V_{u s}\right|=\lambda=0.2240 \pm 0.0036 \quad\left|V_{c b}\right|=(41.5 \pm 0.8) \cdot 10^{-3}, \\
& \frac{\left|V_{u b}\right|}{\left|V_{c b}\right|}=0.086 \pm 0.008, \quad\left|V_{u b}\right|=(3.57 \pm 0.31) \cdot 10^{-3} .
\end{aligned}
$$

implying

$$
A=0.83 \pm 0.02, \quad R_{b}=0.37 \pm 0.04 .
$$

There is an impressive work done by theorists and experimentalists hidden behind these numbers. We refer to [3] for details. See also [19].

The information given above tells us only that the apex $A$ of the unitarity triangle lies in the band shown in fig. 22 While this information appears at first sight to be rather limited, it is very important for the following reason. As $\left|V_{u s}\right|,\left|V_{c b}\right|,\left|V_{u b}\right|$ and consequently $R_{b}$ are determined here from tree level decays, their values given above are to an excellent accuracy independent of any new physics contributions. They are universal fundamental constants valid in any extention of the SM. Therefore their precise determinations are of utmost importance. In order to answer the question where the apex $A$ lies on

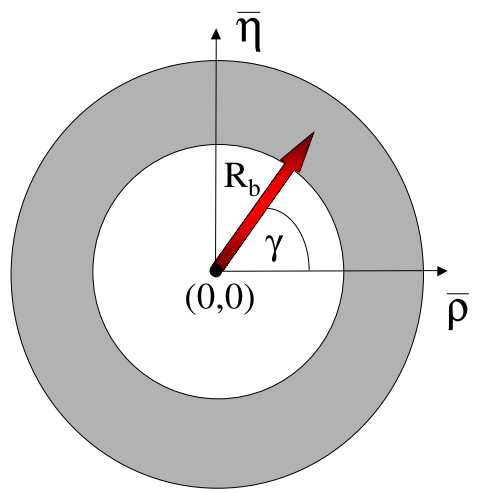

Fig. 2. "Unitarity Clock".

the "unitarity clock" in fig. 2 we have to look at other decays. Most promising in this respect are the so-called "loop induced" decays and transitions and CP-violating B decays. These decays are sensitive to the angles $\beta$ and $\gamma$ as well as to the length $R_{t}$ and measuring only one of these three quantities allows to find the unitarity triangle provided the universal $R_{b}$ is known.

Of course any pair among $\left(R_{t}, \beta, \gamma\right)$ is sufficient to construct the UT without any knowledge of $R_{b}$. Yet the special role of $R_{b}$ among these variables 
lies in its universality whereas the other three variables are generally sensitive functions of possible new physics contributions. This means that assuming three generation unitarity of the CKM matrix and that the SM is a part of a bigger theory, the apex of the unitarity triangle has to be eventually placed on the unitarity clock with the radius $R_{b}$ obtained from tree level decays. That is even if using SM expressions for loop induced processes, $(\bar{\varrho}, \bar{\eta})$ would be found outside the unitarity clock, the corresponding expressions of the grander theory must include appropriate new contributions so that the apex of the unitarity triangle is shifted back to the band in fig. 2. In the case of $\mathrm{CP}$ asymmetries this could be achieved by realizing that the measured angles $\alpha$, $\beta$ and $\gamma$ are not the true angles of the unitarity triangle but sums of the true angles and new complex phases present in extentions of the SM. The better $R_{b}$ is known, the thiner the band in fig. 2 will be, selecting in this manner efficiently the correct theory. On the other hand as the the branching ratios for rare and CP-violating decays depend sensitively on the parameter $A$, the precise knowledge of $\left|V_{c b}\right|$ is also very important.

\subsection{Grand Picture}

The apex $(\bar{\varrho}, \bar{\eta})$ of the UT can be efficiently hunted by means of rare and CP violating transitions as shown in fig. 3. Moreover the angles of this triangle can be measured in CP asymmetries in B-decays and using other strategies. This picture could describe in principle the reality in the year 2012, my retirement year, if the SM is the whole story. On the other hand in the presence of significant new physics contributions, the use of the SM expressions for rare and $\mathrm{CP}$ violating transitions in question, combined with future precise measurements, may result in curves which do not cross each other at a single point in the $(\bar{\varrho}, \bar{\eta})$ plane. This would be truly exciting and most of us hope that this will turn out to be the case. In order to be able to draw such thin curves as in fig. 3 not only experiments but also the theory has to be under control.

\subsection{Theoretical Framework}

The present framework for weak decays is based on the operator product expansion (OPE) that allows to separate short and long distance contributions to weak amplitudes and on the renormalization group (RG) methods that allow to sum large logarithms $\log \mu_{S D} / \mu_{L D}$ to all orders in perturbation theory. The full exposition of these methods can be found in [4, 8].

The OPE allows to write the effective weak Hamiltonian simply as follows

$$
\mathcal{H}_{\text {eff }}=\frac{G_{F}}{\sqrt{2}} \sum_{i} V_{\mathrm{CKM}}^{i} C_{i}(\mu) Q_{i} .
$$

Here $G_{F}$ is the Fermi constant and $Q_{i}$ are the relevant local operators which govern the decays in question. They are built out of quark and lepton fields. 


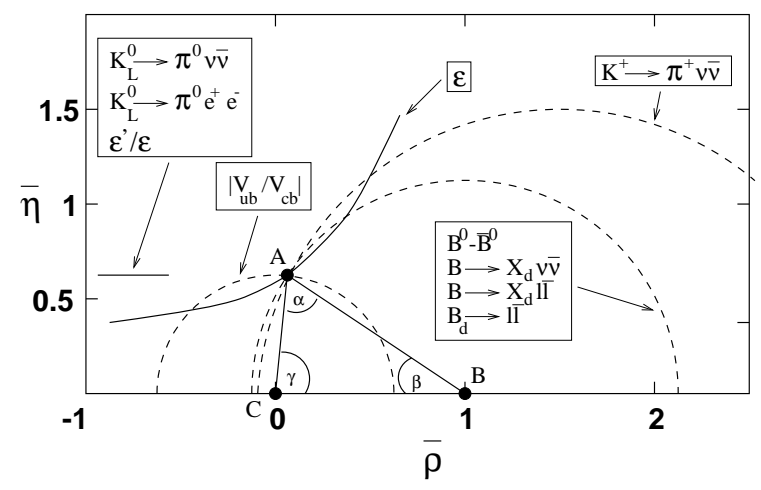

Fig. 3. The ideal Unitarity Triangle.

The Cabibbo-Kobayashi-Maskawa factors $V_{\mathrm{CKM}}^{i}$ [1] 2] and the Wilson coefficients $C_{i}(\mu)$ describe the strength with which a given operator enters the Hamiltonian. The latter coefficients can be considered as scale dependent "couplings" related to "vertices" $Q_{i}$ and as discussed below can be calculated using perturbative methods as long as $\mu$ is not too small.

An amplitude for a decay of a given meson $M=K, B, .$. into a final state $F=\pi \nu \bar{\nu}, \pi \pi, D K$ is then simply given by

$$
A(M \rightarrow F)=\left\langle F\left|\mathcal{H}_{e f f}\right| M\right\rangle=\frac{G_{F}}{\sqrt{2}} \sum_{i} V_{C K M}^{i} C_{i}(\mu)\left\langle F\left|Q_{i}(\mu)\right| M\right\rangle,
$$

where $\left\langle F\left|Q_{i}(\mu)\right| M\right\rangle$ are the matrix elements of $Q_{i}$ between $\mathrm{M}$ and $\mathrm{F}$, evaluated at the renormalization scale $\mu$.

The essential virtue of OPE is this one. It allows to separate the problem of calculating the amplitude $A(M \rightarrow F)$ into two distinct parts: the short distance (perturbative) calculation of the coefficients $C_{i}(\mu)$ and the long-distance (generally non-perturbative) calculation of the matrix elements $\left\langle Q_{i}(\mu)\right\rangle$. The scale $\mu$ separates, roughly speaking, the physics contributions into short distance contributions contained in $C_{i}(\mu)$ and the long distance contributions contained in $\left\langle Q_{i}(\mu)\right\rangle$. Thus $C_{i}$ include the top quark contributions and contributions from other heavy particles such as W-, Z-bosons and charged Higgs particles or supersymmetric particles in the supersymmetric extensions of the SM. Consequently $C_{i}(\mu)$ depend generally on $m_{t}$ and also on the masses of new particles if extensions of the SM are considered. This dependence can be found by evaluating so-called box and penguin diagrams with full W-, Z-, top- and new particles exchanges and properly including short distance QCD effects. The latter govern the $\mu$-dependence of $C_{i}(\mu)$.

The value of $\mu$ can be chosen arbitrarily but the final result must be $\mu$-independent. Therefore the $\mu$-dependence of $C_{i}(\mu)$ has to cancel the $\mu$ - 
dependence of $\left\langle Q_{i}(\mu)\right\rangle$. The same comments apply to the renormalization scheme dependence of $C_{i}(\mu)$ and $\left\langle Q_{i}(\mu)\right\rangle$.

Now due to the fact that for low energy processes the appropriate scale $\mu \ll$ $M_{W, Z}, m_{t}$, large logarithms $\ln M_{\mathrm{W}} / \mu$ compensate in the evaluation of $C_{i}(\mu)$ the smallness of the QCD coupling constant $\alpha_{s}$ and terms $\alpha_{s}^{n}\left(\ln M_{\mathrm{W}} / \mu\right)^{n}$, $\alpha_{s}^{n}\left(\ln M_{\mathrm{W}} / \mu\right)^{n-1}$ etc. have to be resummed to all orders in $\alpha_{s}$ before a reliable result for $C_{i}$ can be obtained. This can be done very efficiently by means of the renormalization group methods. The resulting renormalization group improved perturbative expansion for $C_{i}(\mu)$ in terms of the effective coupling constant $\alpha_{s}(\mu)$ does not involve large logarithms and is more reliable. The related technical issues are discussed in detail in [4] and [8]. It should be emphasized that by 2003 the next-to-leading (NLO) QCD and QED corrections to all relevant weak decay processes in the SM are known.

Clearly, in order to calculate the amplitude $A(M \rightarrow F)$ the matrix elements $\left\langle Q_{i}(\mu)\right\rangle$ have to be evaluated. Since they involve long distance contributions one is forced in this case to use non-perturbative methods such as lattice calculations, the $1 / \mathrm{N}$ expansion ( $\mathrm{N}$ is the number of colours), $\mathrm{QCD}$ sum rules, hadronic sum rules and chiral perturbation theory. In the case of B-meson decays, the Heavy Quark Effective Theory (HQET) and Heavy Quark Expansions (HQE) also turn out to be useful tools. However, all these non-perturbative methods have some limitations. Consequently the dominant theoretical uncertainties in the decay amplitudes reside in the matrix elements $\left\langle Q_{i}(\mu)\right\rangle$ and non-perturbative parameters present in HQET and HQE. These issues are reviewed in $[3]$.

The fact that in many cases the matrix elements $\left\langle Q_{i}(\mu)\right\rangle$ cannot be reliably calculated at present, is very unfortunate. The main goals of the experimental studies of weak decays is the determination of the CKM factors $V_{\mathrm{CKM}}$ and the search for the physics beyond the SM. Without a reliable estimate of $\left\langle Q_{i}(\mu)\right\rangle$ these goals cannot be achieved unless these matrix elements can be determined experimentally or removed from the final measurable quantities by taking suitable ratios and combinations of decay amplitudes or branching ratios. We will encounter many examples in these lectures. Flavour symmetries like $S U(2)_{\mathrm{F}}$ and $S U(3)_{\mathrm{F}}$ relating various matrix elements can be useful in this respect, provided flavour symmetry breaking effects can be reliably calculated. A recent progress in the calculation of $\left\langle Q_{i}(\mu)\right\rangle$ relevant for non-leptonic B decays can be very helpful here as discussed in Section 5 .

After these general remarks let us be more specific about the structure of (23) by considering the simplest class of models in which all flavour violating and CP-violating transition are governed by the CKM matrix and the only relevant local operators are the ones that are relevant in the SM. We will call this scenario "Minimal Flavour Violation" (MFV) 24] being aware of the fact that for some authors MFV means a more general framework in which also new operators can give significant contributions. See for instance the recent discussions in 25] 26]. In the MFV models, as defined in 24], the formula (23) can be written as follows 


$$
\mathrm{A}(\text { Decay })=\sum_{i} B_{i} \eta_{\mathrm{QCD}}^{i} V_{\mathrm{CKM}}^{i} F^{i}, \quad F^{i}=F_{\mathrm{SM}}^{i}+F_{\text {New }}^{i}
$$

with $F_{\mathrm{SM}}^{i}$ and $F_{\text {New }}^{i}$ being real.

Here the non-perturbative parameters $B_{i}$ represent the matrix elements of local operators present in the SM. For instance in the case of $K^{0}-\bar{K}^{0}$ mixing, the matrix element of the operator $\bar{s} \gamma_{\mu}\left(1-\gamma_{5}\right) d \otimes \bar{s} \gamma^{\mu}\left(1-\gamma_{5}\right) d$ is represented by the parameter $\hat{B}_{K}$. There are other non-perturbative parameters in the SM that represent matrix elements of operators $Q_{i}$ with different colour and Dirac structures. The objects $\eta_{\mathrm{QCD}}^{i}$ are the QCD factors resulting from RG-analysis of the corresponding operators and $F_{\mathrm{SM}}^{i}$ stand for the so-called Inami-Lim functions [27. that result from the calculations of various box and penguin diagrams. They depend on the top-quark mass. $V_{\text {CKM }}^{i}$ are the CKM-factors we want to determine.

The important point is that in all MFV models $B_{i}$ and $\eta_{\mathrm{QCD}}^{i}$ are the same as in the SM and the only place where the new physics enters are the new short distance functions $F_{\text {New }}^{i}$ that depend on the new parameters in the extensions of the SM like the masses of charginos, squarks, charged Higgs particles and $\tan \beta=v_{2} / v_{1}$ in the MSSM. These new particles enter the new box and penguin diagrams. Strictly speaking at the NLO level the QCD corrections to the new diagrams at scales larger than $\mathcal{O}\left(M_{\mathrm{W}}\right)$ may differ from the corresponding corrections in the SM but this, generally small, difference can be absorbed into $F_{\mathrm{New}}^{i}$ so that $\eta_{\mathrm{QCD}}^{i}$ are the QCD corrections calculated in the SM. Indeed, the QCD corrections at scales lower than $\mathcal{O}\left(M_{\mathrm{W}}\right)$ are related to the renormalization of the local operators that are common to all models in this class.

In more complicated extensions of the SM new operators (Dirac structures) that are either absent or very strongly suppressed in the SM, can become important. Moreover new sources of flavour and $\mathrm{CP}$ violation beyond the CKM matrix, including new complex phases, could be present. A general master formula describing such contributions is given in [28].

Finally, let me give some arguments why our definition of MFV models is phenomenologically useful. With a simple formula like (24) it is possible to derive a number of relations that are independent of the parameters specific to a given MFV models. Consequently, any violation of these relations will signal the presence of new local operators and/or new complex phases that are necessary to describe the data. We will return to this point in Section 7 .

\section{Particle-Antiparticle Mixing and Various Types of CP Violation}

\subsection{Preliminaries}

Let us next discuss the formalism of particle-antiparticle mixing and $\mathrm{CP}$ violation. Much more elaborate discussion can be found in two books [11 12]. 
We will concentrate here on $K^{0}-\bar{K}^{0}$ mixing, $B_{d, s}^{0}-\bar{B}_{d, s}^{0}$ mixings and CP violation in K-meson and B-meson decays. Due to GIM mechanism 29] the phenomena discussed in this section appear first at the one-loop level and as such they are sensitive measures of the top quark couplings $V_{t i}(i=d, s, b)$ and in particular of the phase $\delta=\gamma$. They allow then to construct the unitarity triangle as explicitly demonstrated in Section 4 .

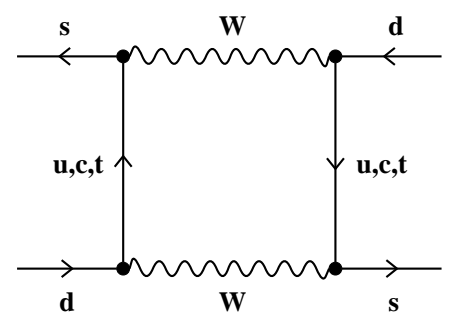

(a)

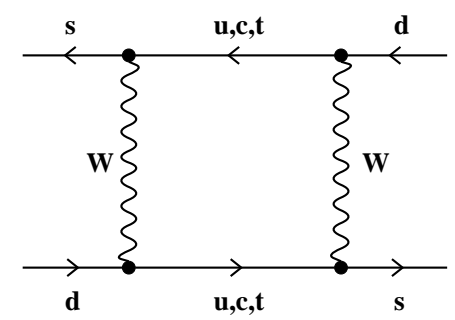

(b)

Fig. 4. Box diagrams contributing to $K^{0}-\bar{K}^{0}$ mixing in the SM.

\subsection{Express Review of $K^{0}-\bar{K}^{0}$ Mixing}

$K^{0}=(\bar{s} d)$ and $\bar{K}^{0}=(s \bar{d})$ are flavour eigenstates which in the SM may mix via weak interactions through the box diagrams in fig. 团 We will choose the phase conventions so that

$$
C P\left|K^{0}\right\rangle=-\left|\bar{K}^{0}\right\rangle, \quad C P\left|\bar{K}^{0}\right\rangle=-\left|K^{0}\right\rangle .
$$

In the absence of mixing the time evolution of $\left|K^{0}(t)\right\rangle$ is given by

$$
\left|K^{0}(t)\right\rangle=\left|K^{0}(0)\right\rangle \exp (-i H t), \quad H=M-i \frac{\Gamma}{2},
$$

where $M$ is the mass and $\Gamma$ the width of $K^{0}$. Similar formula exists for $\bar{K}^{0}$.

On the other hand, in the presence of flavour mixing the time evolution of the $K^{0}-\bar{K}^{0}$ system is described by

$$
i \frac{d \psi(t)}{d t}=\hat{H} \psi(t) \quad \psi(t)=\left(\begin{array}{l}
\left|K^{0}(t)\right\rangle \\
\left|\bar{K}^{0}(t)\right\rangle
\end{array}\right)
$$

where

$$
\hat{H}=\hat{M}-i \frac{\hat{\Gamma}}{2}=\left(\begin{array}{l}
M_{11}-i \frac{\Gamma_{11}}{2} M_{12}-i \frac{\Gamma_{12}}{2} \\
M_{21}-i \frac{I_{21}}{2} M_{22}-i \frac{\Gamma_{22}}{2}
\end{array}\right)
$$

with $\hat{M}$ and $\hat{\Gamma}$ being hermitian matrices having positive (real) eigenvalues in analogy with $M$ and $\Gamma . M_{i j}$ and $\Gamma_{i j}$ are the transition matrix elements from virtual and physical intermediate states respectively. Using 


$$
\begin{aligned}
& M_{21}=M_{12}^{*}, \quad \Gamma_{21}=\Gamma_{12}^{*}, \quad \text { (hermiticity) } \\
& M_{11}=M_{22} \equiv M, \quad \Gamma_{11}=\Gamma_{22} \equiv \Gamma, \quad(\mathrm{CPT})
\end{aligned}
$$

we have

$$
\hat{H}=\left(\begin{array}{cc}
M-i \frac{\Gamma}{2} & M_{12}-i \frac{\Gamma_{12}}{2} \\
M_{12}^{*}-i \frac{\Gamma_{12}^{*}}{2} & M-i \frac{\Gamma}{2}
\end{array}\right) .
$$

Diagonalizing (27) we find:

\section{Eigenstates:}

$$
K_{L, S}=\frac{(1+\bar{\varepsilon}) K^{0} \pm(1-\bar{\varepsilon}) \bar{K}^{0}}{\sqrt{2\left(1+|\bar{\varepsilon}|^{2}\right)}}
$$

where $\bar{\varepsilon}$ is a small complex parameter given by

$$
\frac{1-\bar{\varepsilon}}{1+\bar{\varepsilon}}=\sqrt{\frac{M_{12}^{*}-i \frac{1}{2} \Gamma_{12}^{*}}{M_{12}-i \frac{1}{2} \Gamma_{12}}}=\frac{2 M_{12}^{*}-i \Gamma_{12}^{*}}{\Delta M-i \frac{1}{2} \Delta \Gamma} \equiv r \exp (i \kappa) .
$$

with $\Delta \Gamma$ and $\Delta M$ given below.

\section{Eigenvalues:}

$$
M_{L, S}=M \pm \operatorname{Re} Q \quad \Gamma_{L, S}=\Gamma \mp 2 \operatorname{Im} Q
$$

where

$$
Q=\sqrt{\left(M_{12}-i \frac{1}{2} \Gamma_{12}\right)\left(M_{12}^{*}-i \frac{1}{2} \Gamma_{12}^{*}\right)} .
$$

Consequently we have

$$
\Delta M=M_{L}-M_{S}=2 \operatorname{Re} Q, \quad \Delta \Gamma=\Gamma_{L}-\Gamma_{S}=-4 \operatorname{Im} Q .
$$

It should be noted that the mass eigenstates $K_{S}$ and $K_{L}$ differ from CP eigenstates

$$
\begin{array}{ll}
K_{1}=\frac{1}{\sqrt{2}}\left(K^{0}-\bar{K}^{0}\right), & C P\left|K_{1}\right\rangle=\left|K_{1}\right\rangle, \\
K_{2}=\frac{1}{\sqrt{2}}\left(K^{0}+\bar{K}^{0}\right), & C P\left|K_{2}\right\rangle=-\left|K_{2}\right\rangle,
\end{array}
$$

by a small admixture of the other CP eigenstate:

$$
K_{\mathrm{S}}=\frac{K_{1}+\bar{\varepsilon} K_{2}}{\sqrt{1+|\bar{\varepsilon}|^{2}}}, \quad K_{\mathrm{L}}=\frac{K_{2}+\bar{\varepsilon} K_{1}}{\sqrt{1+|\bar{\varepsilon}|^{2}}} .
$$

Since $\bar{\varepsilon}$ is $\mathcal{O}\left(10^{-3}\right)$, one has to a very good approximation:

$$
\Delta M_{K}=2 \operatorname{Re} M_{12}, \quad \Delta \Gamma_{K}=2 \operatorname{Re} \Gamma_{12},
$$

where we have introduced the subscript $K$ to stress that these formulae apply only to the $K^{0}-\bar{K}^{0}$ system.

The $K_{\mathrm{L}}-K_{\mathrm{S}}$ mass difference is experimentally measured to be [19] 


$$
\Delta M_{K}=M\left(K_{\mathrm{L}}\right)-M\left(K_{\mathrm{S}}\right)=(3.490 \pm 0.006) \cdot 10^{-15} \mathrm{GeV} .
$$

In the SM roughly $80 \%$ of the measured $\Delta M_{K}$ is described by the real parts of the box diagrams with charm quark and top quark exchanges, whereby the contribution of the charm exchanges is by far dominant. The remaining $20 \%$ of the measured $\Delta M_{K}$ is attributed to long distance contributions which are difficult to estimate [30. Further information with the relevant references can be found in 31. The situation with $\Delta \Gamma_{K}$ is rather different. It is fully dominated by long distance effects. Experimentally one has $\Delta \Gamma_{K} \approx-2 \Delta M_{K}$.

Generally to observe $\mathrm{CP}$ violation one needs an interference between various amplitudes that carry complex phases. As these phases are obviously convention dependent, the $\mathrm{CP}$-violating effects depend only on the differences of these phases. In particular the parameter $\bar{\varepsilon}$ depends on the phase convention chosen for $K^{0}$ and $\bar{K}^{0}$. Therefore it may not be taken as a physical measure of $\mathrm{CP}$ violation. On the other hand $\operatorname{Re} \bar{\varepsilon}$ and $r$, defined in (33) are independent of phase conventions. In fact the departure of $r$ from 1 measures CP violation in the $K^{0}-\bar{K}^{0}$ mixing:

$$
r=1+\frac{2\left|\Gamma_{12}\right|^{2}}{4\left|M_{12}\right|^{2}+\left|\Gamma_{12}\right|^{2}} \operatorname{Im}\left(\frac{M_{12}}{\Gamma_{12}}\right) \approx 1-\operatorname{Im}\left(\frac{\Gamma_{12}}{M_{12}}\right) .
$$

This type of $\mathrm{CP}$ violation can be best isolated in semi-leptonic decays of the $K_{L}$ meson. The non-vanishing asymmetry $a_{\mathrm{SL}}\left(K_{L}\right)$ :

$$
\frac{\Gamma\left(K_{L} \rightarrow \pi^{-} e^{+} \nu_{e}\right)-\Gamma\left(K_{L} \rightarrow \pi^{+} e^{-} \bar{\nu}_{e}\right)}{\Gamma\left(K_{L} \rightarrow \pi^{-} e^{+} \nu_{e}\right)+\Gamma\left(K_{L} \rightarrow \pi^{+} e^{-} \bar{\nu}_{e}\right)}=\left(\operatorname{Im} \frac{\Gamma_{12}}{M_{12}}\right)_{K}=2 \operatorname{Re} \bar{\varepsilon}
$$

signals this type of $\mathrm{CP}$ violation. Note that $a_{S L}\left(\mathrm{~K}_{\mathrm{L}}\right)$ is determined purely by the quantities related to $K^{0}-\bar{K}^{0}$ mixing. Specifically, it measures the difference between the phases of $\Gamma_{12}$ and $M_{12}$.

That a non-vanishing $a_{\mathrm{SL}}\left(K_{L}\right)$ is indeed a signal of CP violation can also be understood in the following manner. $K_{L}$, that should be a CP eigenstate $K_{2}$ in the case of $\mathrm{CP}$ conservation, decays into $\mathrm{CP}$ conjugate final states with different rates. As $\operatorname{Re} \bar{\varepsilon}>0, K_{L}$ prefers slightly to decay into $\pi^{-} e^{+} \nu_{e}$ than $\pi^{+} e^{-} \bar{\nu}_{e}$. This would not be possible in a CP-conserving world.

\subsection{The First Look at $\varepsilon$ and $\varepsilon^{\prime}$}

Since a two pion final state is $\mathrm{CP}$ even while a three pion final state is $\mathrm{CP}$ odd, $K_{\mathrm{S}}$ and $K_{\mathrm{L}}$ preferably decay to $2 \pi$ and $3 \pi$, respectively via the following $\mathrm{CP}$-conserving decay modes:

$$
K_{\mathrm{L}} \rightarrow 3 \pi \quad\left(\text { via } \mathrm{K}_{2}\right), \quad K_{\mathrm{S}} \rightarrow 2 \pi \quad\left(\text { via } \mathrm{K}_{1}\right) .
$$

This difference is responsible for the large disparity in their life-times. A factor of 579 . However, $K_{\mathrm{L}}$ and $K_{\mathrm{S}}$ are not CP eigenstates and may decay with small branching fractions as follows:

$$
K_{\mathrm{L}} \rightarrow 2 \pi \quad\left(\text { via } \mathrm{K}_{1}\right), \quad K_{\mathrm{S}} \rightarrow 3 \pi \quad\left(\text { via } \mathrm{K}_{2}\right) .
$$


This violation of $\mathrm{CP}$ is called indirect as it proceeds not via explicit breaking of the CP symmetry in the decay itself but via the admixture of the CP state with opposite CP parity to the dominant one. The measure for this indirect $\mathrm{CP}$ violation is defined as $(\mathrm{I}=\mathrm{isospin})$

$$
\varepsilon \equiv \frac{A\left(K_{\mathrm{L}} \rightarrow(\pi \pi)_{I=0}\right)}{A\left(K_{\mathrm{S}} \rightarrow(\pi \pi)_{I=0}\right)} .
$$

Following the derivation in 32 one finds

$$
\varepsilon=\bar{\varepsilon}+i \xi=\frac{\exp (i \pi / 4)}{\sqrt{2} \Delta M_{K}}\left(\operatorname{Im} M_{12}+2 \xi \operatorname{Re} M_{12}\right), \quad \xi=\frac{\operatorname{Im} A_{0}}{\operatorname{Re} A_{0}} .
$$

The phase convention dependence of $\xi$ cancells the one of $\bar{\varepsilon}$ so that $\varepsilon$ is free from this dependence. The isospin amplitude $A_{0}$ is defined below.

The important point in the definition (46) is that only the transition to $(\pi \pi)_{I=0}$ enters. The transition to $(\pi \pi)_{I=2}$ is absent. This allows to remove a certain type of $\mathrm{CP}$ violation that originates in decays only. Yet as $\varepsilon \neq \bar{\varepsilon}$ and only $\operatorname{Re} \varepsilon=\operatorname{Re} \bar{\varepsilon}$, it is clear that $\varepsilon$ includes a type of $\mathrm{CP}$ violation represented by $\operatorname{Im} \varepsilon$ which is absent in the semileptonic asymmetry (43). We will identify this type of $\mathrm{CP}$ violation in Section 2.7, where a more systematic classification of different types of $\mathrm{CP}$ violation will be given.

While indirect $\mathrm{CP}$ violation reflects the fact that the mass eigenstates are not $\mathrm{CP}$ eigenstates, so-called direct $\mathrm{CP}$ violation is realized via a direct transition of a $\mathrm{CP}$ odd to a $\mathrm{CP}$ even state: $K_{2} \rightarrow \pi \pi$. A measure of such a direct $\mathrm{CP}$ violation in $K_{L} \rightarrow \pi \pi$ is characterized by a complex parameter $\varepsilon^{\prime}$ defined as

$$
\varepsilon^{\prime} \equiv \frac{1}{\sqrt{2}}\left(\frac{A_{2, L}}{A_{0, S}}-\frac{A_{2, S}}{A_{0, S}} \frac{A_{0, L}}{A_{0, S}}\right)
$$

where $A_{I, L} \equiv A\left(K_{L} \rightarrow(\pi \pi)_{I}\right)$ and $A_{I, S} \equiv A\left(K_{S} \rightarrow(\pi \pi)_{I}\right)$.

This time the transitions to $(\pi \pi)_{I=0}$ and $(\pi \pi)_{I=2}$ are included which allows to study $\mathrm{CP}$ violation in the decay itself. We will discuss this issue in general terms in Section 2.7. It is useful to cast (48) into

$$
\varepsilon^{\prime}=\frac{1}{\sqrt{2}} \operatorname{Im}\left(\frac{A_{2}}{A_{0}}\right) \exp \left(i \Phi_{\varepsilon^{\prime}}\right), \quad \Phi_{\varepsilon^{\prime}}=\frac{\pi}{2}+\delta_{2}-\delta_{0},
$$

where the isospin amplitudes $A_{I}$ in $K \rightarrow \pi \pi$ decays are introduced through

$$
\begin{aligned}
& A\left(K^{+} \rightarrow \pi^{+} \pi^{0}\right)=\sqrt{\frac{3}{2}} A_{2} e^{i \delta_{2}}, \\
& A\left(K^{0} \rightarrow \pi^{+} \pi^{-}\right)=\sqrt{\frac{2}{3}} A_{0} e^{i \delta_{0}}+\sqrt{\frac{1}{3}} A_{2} e^{i \delta_{2}}, \\
& A\left(K^{0} \rightarrow \pi^{0} \pi^{0}\right)=\sqrt{\frac{2}{3}} A_{0} e^{i \delta_{0}}-2 \sqrt{\frac{1}{3}} A_{2} e^{i \delta_{2}} .
\end{aligned}
$$


Here the subscript $I=0,2$ denotes states with isospin 0,2 equivalent to $\Delta I=$ $1 / 2$ and $\Delta I=3 / 2$ transitions, respectively, and $\delta_{0,2}$ are the corresponding strong phases. The weak CKM phases are contained in $A_{0}$ and $A_{2}$. The isospin amplitudes $A_{I}$ are complex quantities which depend on phase conventions. On the other hand, $\varepsilon^{\prime}$ measures the difference between the phases of $A_{2}$ and $A_{0}$ and is a physical quantity. The strong phases $\delta_{0,2}$ can be extracted from $\pi \pi$ scattering. Then $\Phi_{\varepsilon^{\prime}} \approx \pi / 4$. See [33] for more details.

Experimentally $\varepsilon$ and $\varepsilon^{\prime}$ can be found by measuring the ratios

$$
\eta_{00}=\frac{A\left(K_{\mathrm{L}} \rightarrow \pi^{0} \pi^{0}\right)}{A\left(K_{\mathrm{S}} \rightarrow \pi^{0} \pi^{0}\right)}, \quad \eta_{+-}=\frac{A\left(K_{\mathrm{L}} \rightarrow \pi^{+} \pi^{-}\right)}{A\left(K_{\mathrm{S}} \rightarrow \pi^{+} \pi^{-}\right)} .
$$

Indeed, assuming $\varepsilon$ and $\varepsilon^{\prime}$ to be small numbers one finds

$$
\eta_{00}=\varepsilon-\frac{2 \varepsilon^{\prime}}{1-\sqrt{2} \omega}, \quad \eta_{+-}=\varepsilon+\frac{\varepsilon^{\prime}}{1+\omega / \sqrt{2}}
$$

where $\omega=\operatorname{Re} A_{2} / \operatorname{Re} A_{0}=0.045$. In the absence of direct $\mathrm{CP}$ violation $\eta_{00}=$ $\eta_{+-}$. The ratio $\varepsilon^{\prime} / \varepsilon$ can then be measured through

$$
\operatorname{Re}\left(\varepsilon^{\prime} / \varepsilon\right)=\frac{1}{6(1+\omega / \sqrt{2})}\left(1-\left|\frac{\eta_{00}}{\eta_{+-}}\right|^{2}\right) .
$$

\subsection{Basic Formula for $\varepsilon$}

With all this information at hand one can derive a formula for $\varepsilon$ which can be efficiently used in pheneomenological applications. As this derivation has been presented in detail in [6], we will be very brief here.

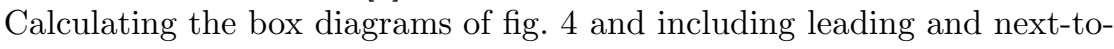
leading QCD corrections one finds

$$
\begin{aligned}
& M_{12}=D_{\varepsilon}\left[\lambda_{c}^{* 2} \eta_{1} S_{0}\left(x_{c}\right)+\lambda_{t}^{* 2} \eta_{2} S_{0}\left(x_{t}\right)+2 \lambda_{c}^{*} \lambda_{t}^{*} \eta_{3} S_{0}\left(x_{c}, x_{t}\right)\right], \\
& D_{\varepsilon}=\frac{G_{\mathrm{F}}^{2}}{12 \pi^{2}} F_{K}^{2} \hat{B}_{K} m_{K} M_{\mathrm{W}}^{2}
\end{aligned}
$$

where $F_{K}=160 \mathrm{MeV}$ is the $K$-meson decay constant and $m_{K}$ the $K$-meson mass. Next, the renormalization group invariant parameter $\hat{B}_{K}$ is defined by

$$
\begin{aligned}
& \hat{B}_{K}=B_{K}(\mu)\left[\alpha_{s}^{(3)}(\mu)\right]^{-2 / 9}\left[1+\frac{\alpha_{s}^{(3)}(\mu)}{4 \pi} J_{3}\right], \\
& \left\langle\bar{K}^{0}\left|(\bar{s} d)_{V-A}(\bar{s} d)_{V-A}\right| K^{0}\right\rangle \equiv \frac{8}{3} B_{K}(\mu) F_{K}^{2} m_{K}^{2}
\end{aligned}
$$

where $\alpha_{s}^{(3)}$ is the strong coupling constant in an effective three flavour theory and $J_{3}=1.895$ in the NDR scheme 34. The CKM factors are given by $\lambda_{i}=V_{i s}^{*} V_{i d}$ and the functions $S_{0}$ by $\left(x_{i}=m_{i}^{2} / M_{\mathrm{W}}^{2}\right)$ 


$$
\begin{aligned}
& S_{0}\left(x_{t}\right)=2.39\left(\frac{m_{\mathrm{t}}}{167 \mathrm{GeV}}\right)^{1.52}, \quad S_{0}\left(x_{c}\right)=x_{c}, \\
& S_{0}\left(x_{c}, x_{t}\right)=x_{c}\left[\ln \frac{x_{t}}{x_{c}}-\frac{3 x_{t}}{4\left(1-x_{t}\right)}-\frac{3 x_{t}^{2} \ln x_{t}}{4\left(1-x_{t}\right)^{2}}\right] .
\end{aligned}
$$

Short-distance NLO QCD effects are described through the correction factors $\eta_{1}, \eta_{2}, \eta_{3}$ [31, 34, 35, 36]:

$$
\eta_{1}=(1.32 \pm 0.32)\left[\frac{1.30 \mathrm{GeV}}{m_{c}\left(m_{c}\right)}\right]^{1.1}, \quad \eta_{2}=0.57 \pm 0.01, \quad \eta_{3}=0.47 \pm 0.05
$$

To proceed further we neglect the last term in (47) as it constitutes at most a $2 \%$ correction to $\varepsilon$. This is justified in view of other uncertainties, in particular those connected with $\hat{B}_{K}$. Inserting (56) into (47) we find

$$
\varepsilon=C_{\varepsilon} \hat{B}_{K} \operatorname{Im} \lambda_{t}\left\{\operatorname{Re} \lambda_{c}\left[\eta_{1} S_{0}\left(x_{c}\right)-\eta_{3} S_{0}\left(x_{c}, x_{t}\right)\right]-\operatorname{Re} \lambda_{t} \eta_{2} S_{0}\left(x_{t}\right)\right\} e^{i \pi / 4},
$$

where the numerical constant $C_{\varepsilon}$ is given by

$$
C_{\varepsilon}=\frac{G_{\mathrm{F}}^{2} F_{K}^{2} m_{K} M_{\mathrm{W}}^{2}}{6 \sqrt{2} \pi^{2} \Delta M_{K}}=3.837 \cdot 10^{4} .
$$

Comparing (63) with the experimental value for $\varepsilon[19$

$$
\varepsilon_{\text {exp }}=(2.280 \pm 0.013) \cdot 10^{-3} \exp i \Phi_{\varepsilon}, \quad \Phi_{\varepsilon}=\frac{\pi}{4},
$$

one obtains a constraint on the unitarity triangle in fig. 11 See Section 3.

\subsection{Express Review of $B_{d, s}^{0}-\bar{B}_{d, s}^{0}$ Mixing}

The flavour eigenstates in this case are

$$
B_{d}^{0}=(\bar{b} d), \quad \bar{B}_{d}^{0}=(b \bar{d}), \quad B_{s}^{0}=(\bar{b} s), \quad \bar{B}_{s}^{0}=(b \bar{s}) .
$$

They mix via the box diagrams in fig. 4 with $s$ replaced by $b$ in the case of $B_{d}^{0}-\bar{B}_{d}^{0}$ mixing. In the case of $B_{s}^{0}-\bar{B}_{s}^{0}$ mixing also $d$ has to be replaced by $s$.

Dropping the subscripts $(d, s)$ for a moment, it is customary to denote the mass eigenstates by

$$
\begin{array}{ll}
B_{H}=p B^{0}+q \bar{B}^{0}, & B_{L}=p B^{0}-q \bar{B}^{0}, \\
p=\frac{1+\bar{\varepsilon}_{B}}{\sqrt{2\left(1+\left|\bar{\varepsilon}_{B}\right|^{2}\right)}}, & q=\frac{1-\bar{\varepsilon}_{B}}{\sqrt{2\left(1+\left|\bar{\varepsilon}_{B}\right|^{2}\right)}},
\end{array}
$$

with $\bar{\varepsilon}_{B}$ corresponding to $\bar{\varepsilon}$ in the $K^{0}-\bar{K}^{0}$ system. Here "H" and "L" denote Heavy and Light respectively. As in the $B^{0}-\bar{B}^{0}$ system one has $\Delta \Gamma \ll \Delta M$, it is more suitable to distinguish the mass eigenstates by their masses than the corresponding life-times.

The strength of the $B_{d, s}^{0}-\bar{B}_{d, s}^{0}$ mixings is described by the mass differences

$$
\Delta M_{d, s}=M_{H}^{d, s}-M_{L}^{d, s} \text {. }
$$


In contrast to $\Delta M_{K}$, in this case the long distance contributions are estimated to be very small and $\Delta M_{d, s}$ is very well approximated by the relevant box diagrams. Moreover, due $m_{u, c} \ll m_{t}$ only the top sector is relevant.

$\Delta M_{d, s}$ can be expressed in terms of the off-diagonal element in the neutral B-meson mass matrix by using the formulae developed previously for the $\mathrm{K}$ meson system. One finds

$$
\Delta M_{q}=2\left|M_{12}^{(q)}\right|, \quad \Delta \Gamma_{q}=2 \frac{\operatorname{Re}\left(M_{12} \Gamma_{12}^{*}\right)}{\left|M_{12}\right|} \ll \Delta M_{q}, \quad q=d, s .
$$

These formulae differ from (40) because in the B-system $\Gamma_{12} \ll M_{12}$.

We also have

$$
\frac{q}{p}=\frac{2 M_{12}^{*}-i \Gamma_{12}^{*}}{\Delta M-i \frac{1}{2} \Delta \Gamma}=\frac{M_{12}^{*}}{\left|M_{12}\right|}\left[1-\frac{1}{2} \operatorname{Im}\left(\frac{\Gamma_{12}}{M_{12}}\right)\right]
$$

where higher order terms in the small quantity $\Gamma_{12} / M_{12}$ have been neglected. As $\operatorname{Im}\left(\Gamma_{12} / M_{12}\right)<\mathcal{O}\left(10^{-3}\right)$,

- The semileptonic asymmetry $a_{\mathrm{SL}}(B)$ discussed a few pages below is even smaller than $a_{\mathrm{SL}}\left(K_{L}\right)$. Typically $\mathcal{O}\left(10^{-4}\right)$. These are bad news.

- The ratio $q / p$ is a pure phase to an excellent approximation. These are very good news as we will see below.

Inspecting the relevant box diagrams we find

$$
\left(M_{12}^{*}\right)_{d} \propto\left(V_{t d} V_{t b}^{*}\right)^{2}, \quad\left(M_{12}^{*}\right)_{s} \propto\left(V_{t s} V_{t b}^{*}\right)^{2} .
$$

Now, from Section 1 we know that

$$
V_{t d}=\left|V_{t d}\right| e^{-i \beta}, \quad V_{t s}=-\left|V_{t s}\right| e^{-i \beta_{s}}
$$

with $\beta_{s}=\mathcal{O}\left(10^{-2}\right)$. Consequently to an excellent approximation

$$
\left(\frac{q}{p}\right)_{d, s}=e^{i 2 \phi_{M}^{d, s}}, \quad \phi_{M}^{d}=-\beta, \quad \phi_{M}^{s}=-\beta_{s},
$$

with $\phi_{M}^{d, s}$ given entirely by the weak phases in the CKM matrix.

\subsection{Basic Formulae for $\Delta M_{d, s}$}

The formulae for $\Delta M_{d, s}$ have been derived in [6] with the result

$$
\Delta M_{q}=\frac{G_{\mathrm{F}}^{2}}{6 \pi^{2}} \eta_{B} m_{B_{q}}\left(\hat{B}_{B_{q}} F_{B_{q}}^{2}\right) M_{\mathrm{W}}^{2} S_{0}\left(x_{t}\right)\left|V_{t q}\right|^{2},
$$

where $F_{B_{q}}$ is the $B_{q}$-meson decay constant, $\hat{B}_{q}$ renormalization group invariant parameters defined in analogy to (58) and (59) and $\eta_{B}$ stands for short distance QCD corrections 34, 37.

$$
\eta_{B}=0.55 \pm 0.01 \text {. }
$$

Using (75) we obtain two useful formulae 


$$
\Delta M_{d}=0.50 / \mathrm{ps} \cdot\left[\frac{\sqrt{\hat{B}_{B_{d}}} F_{B_{d}}}{230 \mathrm{MeV}}\right]^{2}\left[\frac{\bar{m}_{\mathrm{t}}\left(m_{\mathrm{t}}\right)}{167 \mathrm{GeV}}\right]^{1.52}\left[\frac{\left|V_{t d}\right|}{7.8 \cdot 10^{-3}}\right]^{2}\left[\frac{\eta_{B}}{0.55}\right]
$$

and

$$
\Delta M_{s}=17.2 / \mathrm{ps} \cdot\left[\frac{\sqrt{\hat{B}_{B_{s}}} F_{B_{s}}}{260 \mathrm{MeV}}\right]^{2}\left[\frac{\bar{m}_{\mathrm{t}}\left(m_{\mathrm{t}}\right)}{167 \mathrm{GeV}}\right]^{1.52}\left[\frac{\left|V_{t s}\right|}{0.040}\right]^{2}\left[\frac{\eta_{B}}{0.55}\right] .
$$

\subsection{Classification of CP Violation}

\section{Preliminaries}

We have mentioned in Section 1 that due to the presence of hadronic matrix elements, various decay amplitudes contain large theoretical uncertainties. It is of interest to investigate which measurements of CP-violating effects do not suffer from hadronic uncertainties. To this end it is useful to make a classification of CP-violating effects that is more transparent than the division into the indirect and direct $\mathrm{CP}$ violation considered so far. A nice detailed presentation has been given by Nir 16 .

Generally complex phases may enter particle-antiparticle mixing and the decay process itself. It is then natural to consider three types of $\mathrm{CP}$ violation:

- $\mathrm{CP}$ Violation in Mixing

- CP Violation in Decay

- CP Violation in the Interference of Mixing and Decay

As the phases in mixing and decay are convention dependent, the CPviolating effects depend only on the differences of these phases. This is clearly seen in the classification given below.

\section{CP Violation in Mixing}

This type of $\mathrm{CP}$ violation can be best isolated in semi-leptonic decays of neutral $\mathrm{B}$ and $\mathrm{K}$ mesons. We have discussed the asymmetry $a_{S L}\left(K_{L}\right)$ before. In the case of $\mathrm{B}$ decays the non-vanishing asymmetry $a_{S L}(B)$ (we suppress the indices $(d, s))$,

$$
\frac{\Gamma\left(\bar{B}^{0}(t) \rightarrow l^{+} \nu X\right)-\Gamma\left(B^{0}(t) \rightarrow l^{-} \bar{\nu} X\right)}{\Gamma\left(\bar{B}^{0}(t) \rightarrow l^{+} \nu X\right)+\Gamma\left(B^{0}(t) \rightarrow l^{-} \bar{\nu} X\right)}=\frac{1-|q / p|^{4}}{1+|q / p|^{4}}=\left(\operatorname{Im} \frac{\Gamma_{12}}{M_{12}}\right)_{B}
$$

signals this type of $\mathrm{CP}$ violation. Here $\bar{B}^{0}(0)=\bar{B}^{0}, B^{0}(0)=B^{0}$. For $t \neq 0$ the formulae analogous to (27) should be used. Note that the final states in (79) contain "wrong charge" leptons and can only be reached in the presence of $B^{0}-\bar{B}^{0}$ mixing. That is one studies effectively the difference between the rates for $\bar{B}^{0} \rightarrow B^{0} \rightarrow l^{+} \nu X$ and $B^{0} \rightarrow \bar{B}^{0} \rightarrow l^{-} \bar{\nu} X$. As the phases in the 
transitions $B^{0} \rightarrow \bar{B}^{0}$ and $\bar{B}^{0} \rightarrow B^{0}$ differ from each other, a non-vanishing $\mathrm{CP}$ asymmetry follows. Specifically $a_{\mathrm{SL}}(B)$ measures the difference between the phases of $\Gamma_{12}$ and $M_{12}$.

As $M_{12}$ and in particular $\Gamma_{12}$ suffer from large hadronic uncertainties, no precise extraction of $\mathrm{CP}$-violating phases from this type of $\mathrm{CP}$ violation can be expected. Moreover as $q / p$ is almost a pure phase, see (71) and (74), the asymmetry is very small and very difficult to measure.

\section{CP Violation in Decay}

This type of $\mathrm{CP}$ violation is best isolated in charged $\mathrm{B}$ and charged $\mathrm{K}$ decays as mixing effects do not enter here. However, it can also be measured in neutral $\mathrm{B}$ and $\mathrm{K}$ decays. The relevant asymmetry is given by

$$
a_{f^{ \pm}}^{\text {decay }}=\frac{\Gamma\left(B^{+} \rightarrow f^{+}\right)-\Gamma\left(B^{-} \rightarrow f^{-}\right)}{\Gamma\left(B^{+} \rightarrow f^{+}\right)+\Gamma\left(B^{-} \rightarrow f^{-}\right)}=\frac{1-\left|\bar{A}_{f^{-}} / A_{f^{+}}\right|^{2}}{1+\left|\bar{A}_{f^{-}} / A_{f^{+}}\right|^{2}}
$$

where

$$
A_{f^{+}}=\left\langle f^{+}\left|\mathcal{H}^{\text {weak }}\right| B^{+}\right\rangle, \quad \bar{A}_{f^{-}}=\left\langle f^{-}\left|\mathcal{H}^{\text {weak }}\right| B^{-}\right\rangle .
$$

For this asymmetry to be non-zero one needs at least two different contributions with different weak $\left(\phi_{i}\right)$ and strong $\left(\delta_{i}\right)$ phases. These could be for instance two tree diagrams, two penguin diagrams or one tree and one penguin. Indeed writing the decay amplitude $A_{f^{+}}$and its CP conjugate $\bar{A}_{f^{-}}$ as

$$
A_{f^{+}}=\sum_{i=1,2} A_{i} e^{i\left(\delta_{i}+\phi_{i}\right)}, \quad \bar{A}_{f^{-}}=\sum_{i=1,2} A_{i} e^{i\left(\delta_{i}-\phi_{i}\right)},
$$

with $A_{i}$ being real, one finds

$$
a_{f^{ \pm}}^{\text {decay }}=\frac{-2 A_{1} A_{2} \sin \left(\delta_{1}-\delta_{2}\right) \sin \left(\phi_{1}-\phi_{2}\right)}{A_{1}^{2}+A_{2}^{2}+2 A_{1} A_{2} \cos \left(\delta_{1}-\delta_{2}\right) \cos \left(\phi_{1}-\phi_{2}\right)} .
$$

The sign of strong phases $\delta_{i}$ is the same for $A_{f^{+}}$and $\bar{A}_{f^{-}}$because CP is conserved by strong interactions. The weak phases have opposite signs.

The presence of hadronic uncertainties in $A_{i}$ and of strong phases $\delta_{i}$ complicates the extraction of the phases $\phi_{i}$ from data. An example of this type of $\mathrm{CP}$ violation in $\mathrm{K}$ decays is $\varepsilon^{\prime}$. We will demonstrate this below.

\section{CP Violation in the Interference of Mixing and Decay}

This type of $\mathrm{CP}$ violation is only possible in neutral $\mathrm{B}$ and $\mathrm{K}$ decays. We will use B decays for illustration suppressing the subscripts $d$ and $s$. Moreover, we set $\Delta \Gamma=0$. Formulae with $\Delta \Gamma \neq 0$ can be found in [9, 16].

Most interesting are the decays into final states which are CP-eigenstates. Then a time dependent asymmetry defined by 


$$
a_{C P}(t, f)=\frac{\Gamma\left(B^{0}(t) \rightarrow f\right)-\Gamma\left(\bar{B}^{0}(t) \rightarrow f\right)}{\Gamma\left(B^{0}(t) \rightarrow f\right)+\Gamma\left(\bar{B}^{0}(t) \rightarrow f\right)}
$$

is given by

$$
a_{C P}(t, f)=a_{C P}^{\text {decay }}(f) \cos (\Delta M t)+a_{C P}^{\text {int }}(f) \sin (\Delta M t)
$$

where we have separated the decay $\mathrm{CP}$-violating contributions from those describing $\mathrm{CP}$ violation in the interference of mixing and decay:

$$
a_{C P}^{\text {decay }}(f)=\frac{1-\left|\xi_{f}\right|^{2}}{1+\left|\xi_{f}\right|^{2}} \equiv C_{f}, \quad a_{C P}^{\text {int }}(f)=\frac{2 \operatorname{Im} \xi_{f}}{1+\left|\xi_{f}\right|^{2}} \equiv-S_{f} .
$$

Here $C_{f}$ and $S_{f}$ are popular notations found in the recent literature. The later type of $\mathrm{CP}$ violation is sometimes called the mixing-induced $\mathrm{CP}$ violation. The quantity $\xi_{f}$ containing all the information needed to evaluate the asymmetries (86) is given by

$$
\xi_{f}=\frac{q}{p} \frac{A\left(\bar{B}^{0} \rightarrow f\right)}{A\left(B^{0} \rightarrow f\right)}=\exp \left(i 2 \phi_{M}\right) \frac{A\left(\bar{B}^{0} \rightarrow f\right)}{A\left(B^{0} \rightarrow f\right)}
$$

with $\phi_{M}$, introduced in (74), denoting the weak phase in the $B^{0}-\bar{B}^{0}$ mixing. $A\left(B^{0} \rightarrow f\right)$ and $A\left(\bar{B}^{0} \rightarrow f\right)$ are decay amplitudes. The time dependence of $a_{C P}(t, f)$ allows to extract $a_{C P}^{\text {decay }}$ and $a_{C P}^{\text {int }}$ as coefficients of $\cos (\Delta M t)$ and $\sin (\Delta M t)$, respectively.

Generally several decay mechanisms with different weak and strong phases can contribute to $A\left(B^{0} \rightarrow f\right)$. These are tree diagram (current-current) contributions, QCD penguin contributions and electroweak penguin contributions. If they contribute with similar strength to a given decay amplitude the resulting CP asymmetries suffer from hadronic uncertainies related to matrix elements of the relevant operators $Q_{i}$. The situation is then analogous to the class just discussed. Indeed

$$
\frac{A\left(\bar{B}^{0} \rightarrow f\right)}{A\left(B^{0} \rightarrow f\right)}=-\eta_{f}\left[\frac{A_{T} e^{i\left(\delta_{T}-\phi_{T}\right)}+A_{P} e^{i\left(\delta_{P}-\phi_{P}\right)}}{A_{T} e^{i\left(\delta_{T}+\phi_{T}\right)}+A_{P} e^{i\left(\delta_{P}+\phi_{P}\right)}}\right]
$$

with $\eta_{f}= \pm 1$ being the CP-parity of the final state, depends on strong phases $\delta_{T, P}$ and hadronic matrix elements present in $A_{T, P}$. Thus the measurement of the asymmetry does not allow a clean determination of the weak phases $\phi_{T, P}$. The minus sign in (88) follows from our CP phase convention $C P\left|B^{0}\right\rangle=$ $-\left|\bar{B}^{0}\right\rangle$, that has also been used in writing the phase factor in (87). Only $\xi$ is phase convention independent. See Section 8.4.1 of 9 for details.

An interesting case arises when a single mechanism dominates the decay amplitude or the contributing mechanisms have the same weak phases. Then the hadronic matrix elements and strong phases drop out and

$$
\frac{A\left(\bar{B}^{0} \rightarrow f\right)}{A\left(B^{0} \rightarrow f\right)}=-\eta_{f} e^{-i 2 \phi_{D}}
$$

is a pure phase with $\phi_{D}$ being the weak phase in $A\left(B^{0} \rightarrow f\right)$. Consequently 


$$
\xi_{f}=-\eta_{f} \exp \left(i 2 \phi_{M}\right) \exp \left(-i 2 \phi_{D}\right), \quad\left|\xi_{f}\right|^{2}=1 .
$$

In this particular case $a_{C P}^{\text {decay }}(f)=C_{f}$ vanishes and the CP asymmetry is given entirely in terms of the weak phases $\phi_{M}$ and $\phi_{D}$ :

$$
a_{C P}(t, f)=\operatorname{Im} \xi_{f} \sin (\Delta M t) \quad \operatorname{Im} \xi_{f}=\eta_{f} \sin \left(2 \phi_{D}-2 \phi_{M}\right)=-S_{f} .
$$

Thus the corresponding measurement of weak phases is free from hadronic uncertainties. A well known example is the decay $B_{d} \rightarrow \psi K_{S}$. Here $\phi_{M}=-\beta$ and $\phi_{D}=0$. As in this case $\eta_{f}=-1$, we find

$$
a_{C P}(t, f)=-\sin (2 \beta) \sin (\Delta M t), \quad S_{f}=\sin (2 \beta)
$$

which allows a very clean measurement of the angle $\beta$ in the unitarity triangle. We will discuss other examples in Section 5.

We observe that the asymmetry $a_{C P}(t, f)$ measures directly the difference between the phases of $B^{0}-\bar{B}^{0}$-mixing $\left(2 \phi_{M}\right)$ and of the decay amplitude $\left(2 \phi_{D}\right)$. This tells us immediately that we are dealing with the interference of mixing and decay. As $\phi_{M}$ and $\phi_{D}$ are phase convention dependent quantities, only their difference is physical, it is impossible to state on the basis of a single asymmetry whether $\mathrm{CP}$ violation takes place in the decay or in the mixing. To this end at least two asymmetries for $B^{0}\left(\bar{B}^{0}\right)$ decays to different final states $f_{i}$ have to be measured. As $\phi_{M}$ does not depend on the final state, $\operatorname{Im} \xi_{f_{1}} \neq \operatorname{Im} \xi_{f_{2}}$ is a signal of CP violation in the decay.

We will see in Section 5 that the ideal situation presented above does not always take place and two or more different mechanism with different weak and strong phases contribute to the CP asymmetry. One finds then

$$
\begin{aligned}
& a_{C P}(t, f)=C_{f} \cos (\Delta M t)-S_{f} \sin (\Delta M t), \\
& C_{f}=-2 r \sin \left(\phi_{1}-\phi_{2}\right) \sin \left(\delta_{1}-\delta_{2}\right) \\
& S_{f}=-\eta_{f}\left[\sin 2\left(\phi_{1}-\phi_{M}\right)+2 r \cos 2\left(\phi_{1}-\phi_{M}\right) \sin \left(\phi_{1}-\phi_{2}\right) \cos \left(\delta_{1}-\delta_{2}\right)\right]
\end{aligned}
$$

where $r=A_{2} / A_{1} \ll 1$ and $\phi_{i}$ and $\delta_{i}$ are weak and strong phases, respectively. For $r=0$ the previous formulae are obtained.

In the case of $\mathrm{K}$ decays, this type of $\mathrm{CP}$ violation can be cleanly measured in the rare decay $K_{L} \rightarrow \pi^{0} \nu \bar{\nu}$. Here the difference between the weak phase in the $K^{0}-\bar{K}^{0}$ mixing and in the decay $\bar{s} \rightarrow \bar{d} \nu \bar{\nu}$ matters.

We can now compare the two classifications of different types of CP violation. $\mathrm{CP}$ violation in mixing is a manifestation of indirect $\mathrm{CP}$ violation. $\mathrm{CP}$ violation in decay is a manifestation of direct $\mathrm{CP}$ violation. $\mathrm{CP}$ violation in interference of mixing and decay contains elements of both the indirect and direct $\mathrm{CP}$ violation.

It is clear from this discussion that only in the case of the third type of $\mathrm{CP}$ violation there are possibilities to measure directly weak phases without hadronic uncertainties and moreover without invoking sophisticated methods. This takes place provided a single mechanism (diagram) is responsible for the decay or the contributing decay mechanisms have the same weak phases. 
However, we will see in Section 5 that there are other strategies, involving also decays to $\mathrm{CP}$ non-eigenstates, that provide clean measurements of the weak phases.

\section{Another Look at $\varepsilon$ and $\varepsilon^{\prime}$}

Let us finally investigate what type of $\mathrm{CP}$ violation is represented by $\varepsilon$ and $\varepsilon^{\prime}$. Here instead of different mechanisms it is sufficient to talk about different isospin amplitudes.

In the case of $\varepsilon, \mathrm{CP}$ violation in decay is not possible as only the isospin amplitude $A_{0}$ is involved. See (46). We know also that only $\operatorname{Re} \varepsilon=\operatorname{Re} \bar{\varepsilon}$ is related to $\mathrm{CP}$ violation in mixing. Consequently:

- Re$\varepsilon$ represents CP violation in mixing,

- $\operatorname{Im} \varepsilon$ represents $\mathrm{CP}$ violation in the interference of mixing and decay.

In order to analyze the case of $\varepsilon^{\prime}$ we use the formula (49) to find

$$
\begin{aligned}
& \operatorname{Re} \varepsilon^{\prime}=-\frac{1}{\sqrt{2}}\left|\frac{A_{2}}{A_{0}}\right| \sin \left(\phi_{2}-\phi_{0}\right) \sin \left(\delta_{2}-\delta_{0}\right) \\
& \operatorname{Im} \varepsilon^{\prime}=\frac{1}{\sqrt{2}}\left|\frac{A_{2}}{A_{0}}\right| \sin \left(\phi_{2}-\phi_{0}\right) \cos \left(\delta_{2}-\delta_{0}\right) .
\end{aligned}
$$

Consequently:

- $\operatorname{Re} \varepsilon^{\prime}$ represents CP violation in decay as it is only non zero provided simultaneously $\phi_{2} \neq \phi_{0}$ and $\delta_{2} \neq \delta_{0}$.

- $\operatorname{Im} \varepsilon^{\prime}$ exists even for $\delta_{2}=\delta_{0}$ but as it requires $\phi_{2} \neq \phi_{0}$ it represents $\mathrm{CP}$ violation in decay as well.

Experimentally $\delta_{2} \neq \delta_{0}$. Within the SM, $\phi_{2}$ and $\phi_{0}$ are connected with electroweak penguins and QCD penguins, respectively. We will see in Section 4 that these phases differ from each other so that a nonvanishing $\varepsilon^{\prime}$ is obtained.

\section{Standard Analysis of the Unitarity Triangle (UT)}

\subsection{General Procedure}

After these general discussion of basic concepts let us concentrate on the standard analysis of the Unitarity Triangle (see fig. (1) within the SM. A very detailed description of this analysis with the participation of the leading experimentalists and theorists in this field can be found in [3].

Setting $\lambda=\left|V_{u s}\right|=0.224$, the analysis proceeds in the following five steps:

Step 1:

From $b \rightarrow c$ transition in inclusive and exclusive leading B-meson decays one finds $\left|V_{c b}\right|$ and consequently the scale of the UT: 
$\left|V_{c b}\right| \Longrightarrow \lambda\left|V_{c b}\right|=\lambda^{3} A$.

Step 2:

From $b \rightarrow u$ transition in inclusive and exclusive $B$ meson decays one finds $\left|V_{u b} / V_{c b}\right|$ and consequently using (12) the side $C A=R_{b}$ of the UT:

$$
\left|\frac{V_{u b}}{V_{c b}}\right| \quad \Longrightarrow \quad R_{b}=\sqrt{\bar{\varrho}^{2}+\bar{\eta}^{2}}=4.35 \cdot\left|\frac{V_{u b}}{V_{c b}}\right| \text {. }
$$

\section{Step 3:}

From the experimental value of $\varepsilon_{K}$ in (65) and the formula (63) rewritten in terms of Wolfenstein parameters one derives the constraint on $(\bar{\varrho}, \bar{\eta})$ 38

$$
\bar{\eta}\left[(1-\bar{\varrho}) A^{2} \eta_{2} S_{0}\left(x_{t}\right)+P_{c}(\varepsilon)\right] A^{2} \hat{B}_{K}=0.187,
$$

where

$$
P_{c}(\varepsilon)=\left[\eta_{3} S_{0}\left(x_{c}, x_{t}\right)-\eta_{1} x_{c}\right] \frac{1}{\lambda^{4}}, \quad x_{i}=\frac{m_{i}^{2}}{M_{\mathrm{W}}^{2}}
$$

with all symbols defined in the previous Section and $P_{c}(\varepsilon)=0.29 \pm 0.07$ 36. summarizing the contributions of box diagrams with two charm quark exchanges and the mixed charm-top exchanges.

As seen in fig. [5 equation (100) specifies a hyperbola in the $(\bar{\varrho}, \bar{\eta})$ plane. The position of the hyperbola depends on $m_{\mathrm{t}},\left|V_{c b}\right|=A \lambda^{2}$ and $\hat{B}_{K}$. With decreasing $m_{\mathrm{t}},\left|V_{c b}\right|$ and $\hat{B}_{K}$ it moves away from the origin of the $(\bar{\varrho}, \bar{\eta})$ plane.

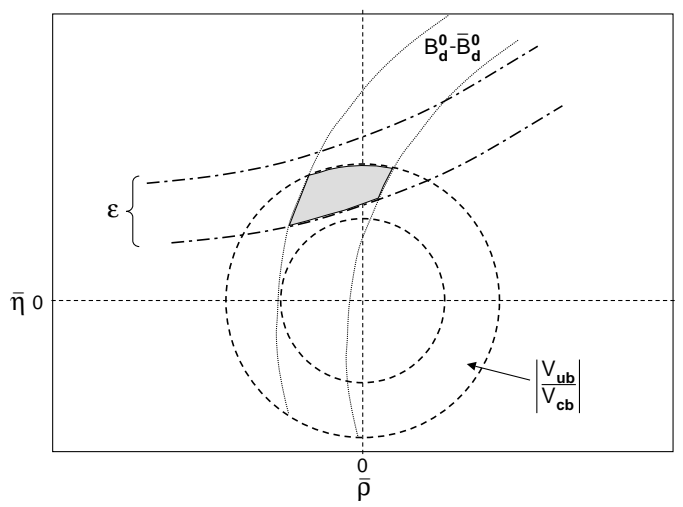

Fig. 5. Schematic determination of the Unitarity Triangle.

\section{Step 4:}

From the measured $\Delta M_{d}$ and the formula (177), the side $A B=R_{t}$ of the UT can be determined:

$$
R_{t}=\frac{1}{\lambda} \frac{\left|V_{t d}\right|}{\left|V_{c b}\right|}=0.85 \cdot\left[\frac{\left|V_{t d}\right|}{7.8 \cdot 10^{-3}}\right]\left[\frac{0.041}{\left|V_{c b}\right|}\right],
$$




$$
\left|V_{t d}\right|=7.8 \cdot 10^{-3}\left[\frac{230 \mathrm{MeV}}{\sqrt{\hat{B}_{B_{d}}} F_{B_{d}}}\right]\left[\frac{167 \mathrm{GeV}}{\bar{m}_{\mathrm{t}}\left(m_{\mathrm{t}}\right)}\right]^{0.76}\left[\frac{\Delta M_{d}}{0.50 / \mathrm{ps}}\right]^{0.5} \sqrt{\frac{0.55}{\eta_{B}}}
$$

with all symbols defined in the previous Section. $\bar{m}_{\mathrm{t}}\left(m_{\mathrm{t}}\right)=(167 \pm 5) \mathrm{GeV}$. Note that $R_{t}$ suffers from additional uncertainty in $\left|V_{c b}\right|$, which is absent in the determination of $\left|V_{t d}\right|$ this way. The constraint in the $(\bar{\varrho}, \bar{\eta})$ plane coming from this step is illustrated in fig. [5]

\section{Step 5:}

The measurement of $\Delta M_{s}$ together with $\Delta M_{d}$ allows to determine $R_{t}$ in a different manner:

$$
R_{t}=0.90\left[\frac{\xi}{1.24}\right] \sqrt{\frac{18.4 / p s}{\Delta M_{s}}} \sqrt{\frac{\Delta M_{d}}{0.50 / p s}}, \quad \xi=\frac{\sqrt{\hat{B}_{B_{s}}} F_{B_{s}}}{\sqrt{\hat{B}_{B_{d}}} F_{B_{d}}} .
$$

One should note that $m_{\mathrm{t}}$ and $\left|V_{c b}\right|$ dependences have been eliminated this way and that $\xi$ should in principle contain much smaller theoretical uncertainties than the hadronic matrix elements in $\Delta M_{d}$ and $\Delta M_{s}$ separately.

The main uncertainties in these steps originate in the theoretical uncertainties in $\hat{B}_{K}$ and $\sqrt{\hat{B}_{d}} F_{B_{d}}$ and to a lesser extent in $\xi[3$ :

$$
\hat{B}_{K}=0.86 \pm 0.15, \quad \sqrt{\hat{B}_{d}} F_{B_{d}}=\left(235_{-41}^{+33}\right) \mathrm{MeV}, \quad \xi=1.24 \pm 0.08 .
$$

Also the uncertainties due to $\left|V_{u b} / V_{c b}\right|$ in step 2 are substantial. The QCD sum rules results for the parameters in question are similar and can be found in 3 . Finally 3

$$
\Delta M_{d}=(0.503 \pm 0.006) / \mathrm{ps}, \quad \Delta M_{s}>14.4 / \mathrm{ps} \text { at } 95 \% \text { C.L. }
$$

\subsection{The Angle $\beta$ from $B_{d} \rightarrow \psi K_{S}$}

One of the highlights of the year 2002 were the considerably improved measurements of $\sin 2 \beta$ by means of the time-dependent CP asymmetry

$$
a_{\psi K_{S}}(t) \equiv-a_{\psi K_{S}} \sin \left(\Delta M_{d} t\right)=-\sin 2 \beta \sin \left(\Delta M_{d} t\right) .
$$

The BaBar 39] and Belle 40] collaborations find

$$
(\sin 2 \beta)_{\psi K_{S}}=\left\{\begin{array}{l}
0.741 \pm 0.067 \text { (stat) } \pm 0.033 \text { (syst) (BaBar) } \\
0.719 \pm 0.074 \text { (stat) } \pm 0.035 \text { (syst) (Belle). }
\end{array}\right.
$$

Combining these results with earlier measurements by $\operatorname{CDF}\left(0.79_{-0.44}^{+0.41}\right)$,

ALEPH $\left(0.84_{-1.04}^{+0.82} \pm 0.16\right)$ and OPAL gives the grand average 41]

$$
(\sin 2 \beta)_{\psi K_{S}}=0.734 \pm 0.054 \text {. }
$$

This is a mile stone in the field of CP violation and in the tests of the SM as we will see in a moment. Not only violation of this symmetry has been confidently established in the B system, but also its size has been measured very accurately. Moreover in contrast to the five constraints listed above, the determination of the angle $\beta$ in this manner is theoretically very clean. 


\subsection{Unitarity Triangle 2003}

We are now in the position to combine all these constraints in order to construct the unitarity triangle and determine various quantities of interest. In this context the important issue is the error analysis of these formulae, in particular the treatment of theoretical uncertainties. In the literature the most popular are the Bayesian approach [42] and the frequentist approach [43]. For the PDG analysis see [19. A critical comparison of these and other methods can be found in [3. I can recommend this reading.

In fig. 6] we show the result of the recent update of an analysis in collaboration with Parodi and Stocchi 44 that uses the Bayesian approach. The results presented below are very close to the ones presented in 3 that was led by my Italian collaborators. The allowed region for $(\bar{\varrho}, \bar{\eta})$ is the area inside the smaller ellipse. We observe that the region $\bar{\varrho}<0$ is disfavoured by the lower bound on $\Delta M_{s}$. It is clear from this figure that the measurement of $\Delta M_{s}$ giving $R_{t}$ through (104) will have a large impact on the plot in fig. 66.

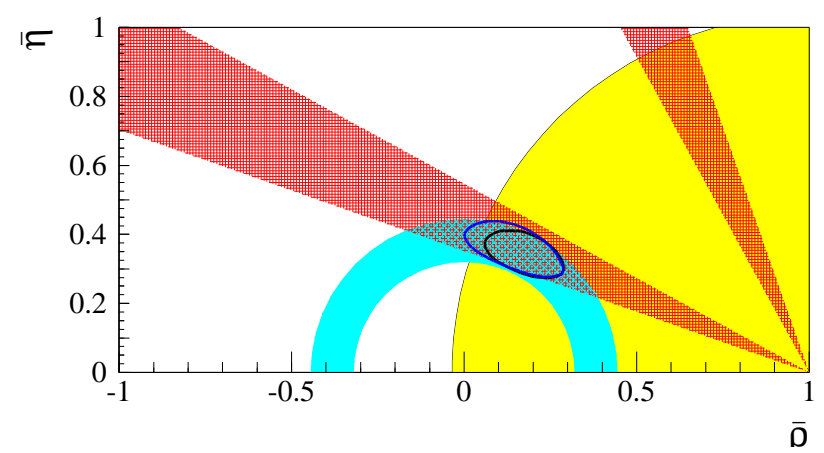

Fig. 6. The allowed $95 \%$ regions in the $(\bar{\varrho}, \bar{\eta})$ plane in the SM (narrower region) and in the MFV models (broader region) from the update of 44]. The individual $95 \%$ regions for the constraint from $\sin 2 \beta, \Delta M_{s}$ and $R_{b}$ are also shown.

The ranges for various quantities that result from this analysis are given in the SM column of table 1 The UUT column will be discussed in Section 7. The SM results follow from the five steps listed above and (108) implying an impressive precision on the angle $\beta$ :

$$
(\sin 2 \beta)_{\text {tot }}=0.705_{-0.032}^{+0.042}, \quad \beta=(22.4 \pm 1.5)^{\circ} .
$$

On the other hand $(\sin 2 \beta)_{\text {ind }}$ obtained by means of the five steps only is found to be 44

$$
(\sin 2 \beta)_{\text {ind }}=0.685 \pm 0.052
$$


demonstrating an excellent agreement (see also fig. (6) between the direct measurement in (108) and the standard analysis of the UT within the SM. This gives a strong indication that the CKM matrix is very likely the dominant source of $\mathrm{CP}$ violation in flavour violating decays. In order to be sure whether this is indeed the case other theoretically clean quantities have to be measured. In particular the angle $\gamma$ that is more sensitive to new physics contributions than $\beta$. We will return to other processes that are useful for the determination of the UT in Sections 5 and 6.

Table 1. Values for different quantities from the update of [4]. $\lambda_{t}=V_{t s}^{*} V_{t d}$.

\begin{tabular}{|c|c|c|}
\hline Strategy & UUT & SM \\
$\bar{\eta}$ & $0.361 \pm 0.032$ & $0.341 \pm 0.028$ \\
$\bar{\varrho}$ & $0.149 \pm 0.056$ & $0.178 \pm 0.046$ \\
$\sin 2 \beta$ & $0.715_{-0.034}^{+0.037}$ & $0.705_{-0.032}^{+0.042}$ \\
$\sin 2 \alpha$ & $0.03 \pm 0.31$ & $-0.19 \pm 0.25$ \\
$\gamma$ & $(67.5 \pm 8.9)^{\circ}$ & $(61.5 \pm 7.0)^{\circ}$ \\
$R_{b}$ & $0.393 \pm 0.025$ & $0.390 \pm 0.024$ \\
$R_{t}$ & $0.925 \pm 0.060$ & $0.890 \pm 0.048$ \\
$\Delta M_{s}\left(p s^{-1}\right)$ & $17.3_{-1.3}^{+2.1}$ & $18.3_{-1.5}^{+1.7}$ \\
$\left|V_{t d}\right|\left(10^{-3}\right)$ & $8.61 \pm 0.55$ & $8.24 \pm 0.41$ \\
$\operatorname{Im} \lambda_{t}\left(10^{-4}\right)$ & $1.39 \pm 0.12$ & $1.31 \pm 0.10$ \\
\hline
\end{tabular}

\section{$4 \varepsilon^{\prime} / \varepsilon$ in the Standard Model}

\subsection{Preliminaries}

The ratio $\varepsilon^{\prime} / \varepsilon$ that parametrizes the size of direct $\mathrm{CP}$ violation with respect to the indirect $\mathrm{CP}$ violation in $K_{L} \rightarrow \pi \pi$ decays has been the subject of very intensive experimental and theoretical studies in the last three decades. After tremendous efforts, on the experimental side the world average based on the recent results from NA48 [45] and $\mathrm{KTeV}$ [46], and previous results from NA31 47] and E731 [8], reads 


$$
\varepsilon^{\prime} / \varepsilon=(16.6 \pm 1.6) \cdot 10^{-4} \quad(2003) .
$$

On the other hand, the theoretical estimates of this ratio are subject to very large hadronic uncertainties. While several analyzes of recent years within the Standard Model (SM) find results that are compatible with (111) 49, 50, 51, 52, 53, 54, 55, 56]), it is fair to say that the chapter on the theoretical calculations of $\varepsilon^{\prime} / \varepsilon$ is far from being closed. A full historical account of the theoretical efforts before 1998 can for example be found in [4, 57. See also [58.

It should be emphasized that all existing analyzes of $\varepsilon^{\prime} / \varepsilon$ use the NLO Wilson coefficients calculated by the Munich and Rome groups in 1993 [59 60, 61] but the hadronic matrix elements, the main theoretical uncertainty in $\varepsilon^{\prime} / \varepsilon$, vary from paper to paper. Nevertheless, apart from the hadronic matrix element of the dominant QCD penguin operator $Q_{6}$, in the last years progress has been made with the determination of all other relevant parameters, which enter the theoretical prediction of $\varepsilon^{\prime} / \varepsilon$. Let me review then briefly the present situation. Further details can be found in 62 .

\subsection{Basic Formulae}

The central formula for $\varepsilon^{\prime} / \varepsilon$ of $49,50,60,62$ can be cast into the following approximate expression (it reproduces the results in 62 to better than $2 \%$ )

$$
\begin{aligned}
& \frac{\varepsilon^{\prime}}{\varepsilon}=\operatorname{Im} \lambda_{t} \cdot F_{\varepsilon^{\prime}}\left(x_{t}\right), \quad \lambda_{t}=V_{t s}^{*} V_{t d}, \\
& F_{\varepsilon^{\prime}}\left(x_{t}\right)=\left[18.7 R_{6}\left(1-\Omega_{\mathrm{IB}}\right)-6.9 R_{8}-1.8\right]\left[\frac{\Lambda_{\overline{\mathrm{MS}}}^{(4)}}{340 \mathrm{MeV}}\right]
\end{aligned}
$$

with the non-perturbative parameters $R_{6}$ and $R_{8}$ defined as

$$
R_{6} \equiv B_{6}^{(1 / 2)}\left[\frac{121 \mathrm{MeV}}{m_{s}\left(m_{c}\right)+m_{d}\left(m_{c}\right)}\right]^{2}, \quad R_{8} \equiv \frac{B_{8}^{(3 / 2)}}{B_{6}^{(1 / 2)}} R_{6} .
$$

The hadronic $B$-parameters $B_{6}^{(1 / 2)}$ and $B_{8}^{(3 / 2)}$ represent the matrix elements of the dominant QCD-penguin $\left(Q_{6}\right)$ and the dominant electroweak penguin $\left(Q_{8}\right)$ operator. In the large $-N_{c}$ approach of 63. they are given by 60, 64]

$$
\begin{aligned}
& \left\langle Q_{6}\right\rangle_{0}=-4 \sqrt{\frac{3}{2}}\left(F_{K}-F_{\pi}\right)\left(\frac{m_{K}^{2}}{121 \mathrm{MeV}}\right)^{2} R_{6}=-0.597 \cdot R_{6} \mathrm{GeV}^{3} \\
& \left\langle Q_{8}\right\rangle_{2}=\sqrt{3} F_{\pi}\left(\frac{m_{K}^{2}}{121 \mathrm{MeV}}\right)^{2} R_{8}=0.948 \cdot R_{8} \mathrm{GeV}^{3} .
\end{aligned}
$$

Finally $\Omega_{\mathrm{IB}}=0.06 \pm 0.0865$ represents isospin breaking correction.

In the strict large $-N_{c}$ limit, $B_{6}^{(1 / 2)}=B_{8}^{(3 / 2)}=1$ and 


$$
\frac{R_{6}}{R_{8}}=1, \quad \frac{\left\langle Q_{6}\right\rangle_{0}}{\left\langle Q_{8}\right\rangle_{2}}=-0.63,
$$

so that there is a one-to-one correspondence between $\varepsilon^{\prime} / \varepsilon$ and $R_{6}=R_{8}$ for fixed values of the remaining parameters. Moreover, only for certain values of $m_{s}\left(m_{c}\right)$ is one able to obtain the experimental value for $\varepsilon^{\prime} / \varepsilon$ [66. Note that once $m_{s}\left(m_{c}\right)$ is known, also $R_{6}, R_{8},\left\langle Q_{6}\right\rangle_{0}$ and $\left\langle Q_{8}\right\rangle_{2}$ are known, but they always satisfy the relations in (117).

\subsection{Numerical Results}

The relevant input parameters are as follows. First

$$
\operatorname{Im} \lambda_{t}=(1.31 \pm 0.10) \cdot 10^{-4}, \quad m_{s}\left(m_{c}\right)=115 \pm 20 \mathrm{MeV}
$$

where the value of $m_{s}$ is an average over recent determinations (see references in 62]). The central value corresponds to $m_{s}(2 \mathrm{GeV})=100 \mathrm{MeV}$.

Concerning $\left\langle Q_{8}\right\rangle_{2}$, in the last years progress has been achieved both in the framework of lattice QCD as well as with analytic methods [55, 56, 67, 68 69, 70. The current status of $\left\langle Q_{8}\right\rangle_{2}$ has been summarized nicely in 69. The most precise determination of $\left\langle Q_{8}\right\rangle_{2}$ comes from the lattice QCD measurement 68, corresponding to $R_{8}=0.81 \pm 0.08$. Several analytic methods give higher results but compatible with it. We will use 62

$$
R_{8}=0.8 \pm 0.2, \quad\left\langle Q_{8}\right\rangle_{2}^{\mathrm{NDR}}\left(m_{c}\right)=(0.76 \pm 0.19) \mathrm{GeV}^{3} .
$$

The situation is less clear concerning $\left\langle Q_{6}\right\rangle_{0}$ but assuming that new physics contributions to $\varepsilon^{\prime} / \varepsilon$ can be neglected one finds from (111)-(113) and (119) that 62

$$
R_{6}=1.15 \pm 0.16, \quad\left\langle Q_{6}\right\rangle_{0}^{\mathrm{NDR}}\left(m_{c}\right)=-(0.69 \pm 0.10) \mathrm{GeV}^{3} .
$$

More generally, the correlation between $R_{8}$ and $R_{6}$ that is implied by the data on $\varepsilon^{\prime} / \varepsilon$ is shown in the spirit of the " $\varepsilon^{\prime} / \varepsilon$-path" of 71 (see also 72,56 ) in figure 7 62. The solid straight line corresponds to the central values of parameters, whereas the short-dashed lines are the uncertainties due to a variation of the input parameters. The vertical long-dashed lines indicate the lattice range for $R_{8}$ 68, whereas the ellipse describes the correlation between $R_{6}$ and $R_{8}$ implied by the data on $\varepsilon^{\prime} / \varepsilon$ when taking into account the more conservative constraint on $R_{8}$ given in (119). The value in (120) corresponds to this ellipse. The full circle and diamond in figure 7 represent the central results of [54 and [56] respectively, that are discussed in detail in 62, and the dashed-dotted line shows the strict large- $N_{c}$ relation $R_{6}=R_{8}$.

In table 2 we show $\varepsilon^{\prime} / \varepsilon$ for specific values of $R_{6}, R_{8}$ and $\Lambda_{\overline{\mathrm{MS}}}^{(4)}$, and in table 3 as a function of $m_{s}\left(m_{c}\right)$ and $\Lambda_{\overline{\mathrm{MS}}}^{(4)}$ obtained in the strict large $-N_{c}$ limit. Here $\operatorname{Im} \lambda_{t}=1.34 \cdot 10^{-4}$. 


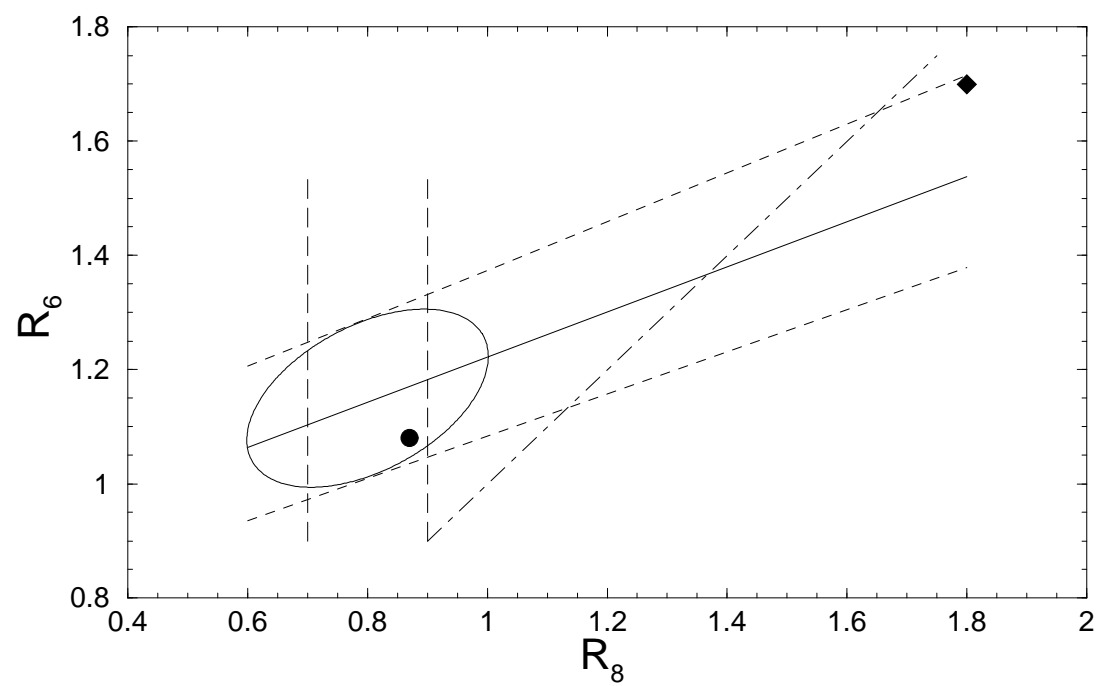

Fig. 7. $R_{6}$ as a function of $R_{8}$. For a detailed explanation see the text.

Table 2. $\varepsilon^{\prime} / \varepsilon$ in units of $10^{-4}$ for $m_{t}=165 \mathrm{GeV}$ and various $\Lambda_{\mathrm{MS}}^{(4)}, R_{6}, R_{8}$.

\begin{tabular}{|c||c|c||c|c||c|c|}
\hline \multicolumn{1}{|c||}{} & \multicolumn{2}{c||}{$R_{6}=1.00$} & \multicolumn{2}{c||}{$R_{6}=1.15$} & \multicolumn{2}{c|}{$R_{6}=1.30$} \\
\hline$\Lambda_{\mathrm{MS}}^{(4)}[\mathrm{MeV}]$ & $R_{8}=0.8$ & $R_{8}=1.0$ & $R_{8}=0.8$ & $R_{8}=1.0$ & $R_{8}=0.8$ & $R_{8}=1.0$ \\
\hline 310 & 12.2 & 10.5 & 15.4 & 13.7 & 18.6 & 16.9 \\
340 & 13.3 & 11.5 & 16.8 & 14.9 & 20.2 & 18.4 \\
370 & 14.6 & 12.6 & 18.3 & 16.3 & 22.0 & 20.0 \\
\hline
\end{tabular}

Table 3. The ratio $\varepsilon^{\prime} / \varepsilon$ in units of $10^{-4}$ for the strict large $-N_{c}$ results $B_{6}^{(1 / 2)}=$ $B_{8}^{(3 / 2)}=1.0, \hat{B}_{K}=0.75$ and various values of $\Lambda_{\overline{\mathrm{MS}}}^{(4)}$ and $m_{s}\left(m_{c}\right)$.

\begin{tabular}{|c||c|c|c|}
\hline$\Lambda_{\overline{\mathrm{MS}}}^{(4)}[\mathrm{MeV}]$ & $m_{s}\left(m_{c}\right)=115 \mathrm{MeV}$ & $m_{s}\left(m_{c}\right)=105 \mathrm{MeV}$ & $m_{s}\left(m_{c}\right)=95 \mathrm{MeV}$ \\
\hline 310 & 10.8 & 13.3 & 16.5 \\
340 & 11.8 & 14.4 & 18.0 \\
370 & 12.9 & 15.8 & 19.6 \\
\hline
\end{tabular}

\subsection{Conclusions}

There are essentially two messages from this analysis [62]

- If indeed $R_{8}=0.8 \pm 0.2$ as indicated by several recent estimates, then the data on $\varepsilon^{\prime} / \varepsilon$ imply

$$
R_{6}=1.15 \pm 0.16, \quad \frac{R_{6}}{R_{8}} \approx 1.4, \quad\left\langle Q_{6}\right\rangle_{0}^{\mathrm{NDR}}\left(m_{c}\right) \approx-\left\langle Q_{8}\right\rangle_{2}^{\mathrm{NDR}}\left(m_{c}\right)
$$


This is in accordance with the results in [54, but differs from the large- $N_{c}$ approach in 63 in which $R_{6} \approx R_{8}$ and $\left\langle Q_{6}\right\rangle_{0}^{\mathrm{NDR}}\left(m_{c}\right)$ is chirally suppressed relatively to $\left\langle Q_{8}\right\rangle_{2}^{\mathrm{NDR}}\left(m_{c}\right)$.

- The large $-N_{c}$ approach of 63 can only be made consistent with data provided

$$
R_{6}=R_{8}=1.36 \pm 0.30
$$

and $\left\langle Q_{8}\right\rangle_{2}^{\mathrm{NDR}}\left(m_{c}\right)$ is higher than obtained by most recent approaches reviewed in 62, 69. This requires $m_{s}\left(m_{c}\right) \leq 105 \mathrm{MeV}$ which is on the low side of (118) but close to low values of $m_{s}\left(m_{c}\right)$ indicated by the most recent lattice simulations with dynamical fermions 73, 74].

Large non-factorizable contributions to $\left\langle Q_{6}\right\rangle_{0}$ and $\left\langle Q_{8}\right\rangle_{2}$ are found in the chiral limit in the large $\mathrm{N}$ approach of [56 and consequently the structure of the matrix elements in question differs in this approach from the formulae (115). Interestingly, in spite of these large non-factorizable contributions, the relation $R_{6}=R_{8}$ is roughly satisfied in this approach (see fig. 17) but one has to go beyond the chiral limit to draw definite conclusions.

As seen in figure 7 all these three scenarios are consistent with the data. Which of these pictures of $\varepsilon^{\prime} / \varepsilon$ is correct, can only be answered by calculating $\left\langle Q_{6}\right\rangle_{0},\left\langle Q_{8}\right\rangle_{2}$ and $m_{s}$ accurately by means of non-perturbative methods that are reliable. Such calculations are independent of the assumption about the role of new physics in $\varepsilon^{\prime} / \varepsilon$ that has been made in [62] in order to extract $\left\langle Q_{6}\right\rangle_{0}$ from the data. If the values for $R_{6,8}$ will be found one day to lie significantly outside the allowed region in figure 7 new physics contributions to $\varepsilon^{\prime} / \varepsilon$ will be required in order to fit the experimental data.

\section{The Angles $\alpha, \beta$ and $\gamma$ from B Decays}

\subsection{Preliminaries}

$\mathrm{CP}$ violation in $\mathrm{B}$ decays is certainly one of the most important targets of $\mathrm{B}$ factories and of dedicated B-experiments at hadron facilities. It is well known that $\mathrm{CP}$-violating effects are expected to occur in a large number of channels at a level attainable experimentally in the near future. Moreover there exist channels which offer the determination of CKM phases essentially without any hadronic uncertainties.

The first results on $\sin 2 \beta$ from BaBar and Belle are very encouraging. These results should be further improved over the coming years through the new measurements of $a_{\psi K_{S}}(t)$ by both collaborations and by CDF and D0 at Fermilab. Moreover measurements of $\mathrm{CP}$ asymmetries in other B decays and the measurements of the angles $\alpha, \beta$ and $\gamma$ by means of various strategies using two-body B decays should contribute substantially to our understanding of $\mathrm{CP}$ violation and will test the $\mathrm{KM}$ picture of $\mathrm{CP}$ violation. 

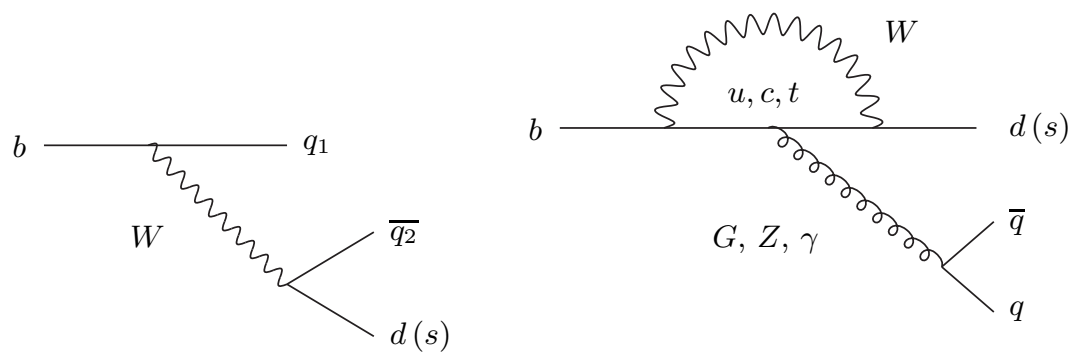

Fig. 8. Tree and penguin diagrams.

The various types of $\mathrm{CP}$ violation have been already classified in Section 2. It turned out that $\mathrm{CP}$ violation in the interference of mixing and decay, in a $\mathrm{B}$ meson decay into a $\mathrm{CP}$ eigenstate, is very suitable for a theoretically clean determination of the angles of the unitarity triangle provided a single CKM phase governs the decay. However as we will see below several useful strategies for the determination of the angles $\alpha, \beta$ and $\gamma$ have been developed that are effective also in the presence of competing CKM phases and when the final state in not a $\mathrm{CP}$ eigenstate. The notes below should only be considered as an introduction to this reach field. For more details the references in Section 1 should be contacted.

\subsection{Classification of Elementary Processes}

Non-leptonic B decays are caused by elementary decays of b quarks that are represented by tree and penguin diagrams in fig. 8 Generally we have

$$
b \rightarrow q_{1} \bar{q}_{2} d(s), \quad b \rightarrow q \bar{q} d(s)
$$

for tree and penguin diagrams, respectively.

There are twelve basic transitions that can be divided into three classes:

Class I: both tree and penguin diagrams contribute. Here $q_{1}=q_{2}=q=$ $u, c$ and consequently the basic transitions are

$$
b \rightarrow c \bar{c} s, \quad b \rightarrow c \bar{c} d, \quad b \rightarrow u \bar{u} s, \quad b \rightarrow u \bar{u} d .
$$

Class II: only tree diagrams contribute. Here $q_{1} \neq q_{2} \in\{u, c\}$ and

$$
b \rightarrow c \bar{u} s, \quad b \rightarrow c \bar{u} d, \quad b \rightarrow u \bar{c} s, \quad b \rightarrow u \bar{c} d .
$$

Class III: only penguin diagrams contribute. Here $q=d, s$ and

$$
b \rightarrow s \bar{s} s, \quad b \rightarrow s \bar{s} d, \quad b \rightarrow d \bar{d} s, \quad b \rightarrow d \bar{d} d .
$$

Now in presenting various decays below, we did not show the corresponding diagrams on purpose. Afterall these are lectures and the exercise for the students is to draw these diagrams by embedding the elementary diagrams of fig. 8 into a given B meson decay. In case of difficulties the student should look at [14, 16] where these diagrams can be found. 


\subsection{Neutral B Decays into CP eigenstates}

\section{$B_{d}^{0} \rightarrow J / \psi K_{S}$ and $\beta$}

The amplitude for this decay can be written as follows

$$
A\left(B_{d}^{0} \rightarrow J / \psi K_{S}\right)=V_{c s} V_{c b}^{*}\left(A_{T}+P_{c}\right)+V_{u s} V_{u b}^{*} P_{u}+V_{t s} V_{t b}^{*} P_{t}
$$

where $A_{T}$ denotes tree diagram contributions and $P_{i}$ with $i=u, c, t$ stand for penguin diagram contributions with internal $u, c$ and $t$ quarks. Now

$$
V_{c s} V_{c b}^{*} \approx A \lambda^{2}, \quad V_{u s} V_{u b}^{*} \approx A \lambda^{4} R_{b} e^{i \gamma}, \quad V_{t s} V_{t b}^{*}=-V_{u s} V_{u b}^{*}-V_{c s} V_{c b}^{*}
$$

with the last relation following from the unitarity of the CKM matrix. Thus

$$
A\left(B_{d}^{0} \rightarrow J / \psi K_{S}\right)=V_{c s} V_{c b}^{*}\left(A_{T}+P_{c}-P_{t}\right)+V_{u s} V_{u b}^{*}\left(P_{u}-P_{t}\right) .
$$

We next note that

$$
\left|\frac{V_{u s} V_{u b}^{*}}{V_{c s} V_{c b}^{*}}\right| \leq 0.02, \quad \frac{P_{u}-P_{t}}{A_{T}+P_{c}-P_{t}} \ll 1
$$

where the last inequality is very plausible as the Wilson coefficients of the current-current operators responsible for $A_{T}$ are much larger than the ones of the penguin operators 4 8. Consequently this decay is dominated by a single CKM factor and as discussed in Section 2, a clean determination of the relevant CKM phase is possible. Indeed in this decay $\phi_{D}=0$ and $\phi_{M}=-\beta$. Using (91) we find then $\left(\eta_{J / \psi K_{S}}=-1\right)$

$$
\begin{aligned}
& a_{C P}^{\mathrm{int}}\left(J / \psi K_{S}\right)=\eta_{J / \psi K_{S}} \sin \left(2 \phi_{D}-2 \phi_{M}\right)=-\sin 2 \beta, \\
& C_{J / \psi K_{S}}=0, \quad S_{J / \psi K_{S}}=\sin 2 \beta
\end{aligned}
$$

that is confirmed by experiment as discussed in Section 3.

$B_{s}^{0} \rightarrow J / \psi \phi$ and $\beta_{s}$

This decay differs from the previous one by the spectator quark, with $d \rightarrow s$ so that the formulae above remain unchanged except that now $\phi_{M}=-\beta_{s}=$ $-\lambda^{2} \bar{\eta}$. A complication arises as the final state is an admixture of $C P=+$ and $C P=-$ states. This issue can be resolved experimentally [14. Choosing $\eta_{J / \psi \phi}=1$ we then find

$$
a_{C P}^{\mathrm{int}}(J / \psi \phi)=\sin \left(2 \phi_{D}-2 \phi_{M}\right)=2 \beta_{s}=2 \lambda^{2} \bar{\eta} \approx 0.03, \quad C_{J / \psi \phi}=0 .(13
$$

Thus this asymmetry measures the phase of $V_{t s}$ that is predicted to be very small from the unitarity of the CKM matrix alone. Because of this there is a lot of room for new physics contributions here. 


\section{$B_{d}^{0} \rightarrow \phi K_{S}$ and $\beta$}

This decay proceeds entirely through penguin diagrams and consequently should be much more sensitive to new physics contributions than the decay $B_{d}^{0} \rightarrow J / \psi K_{S}$. Assuming $\phi=(s \bar{s})$, the decay amplitude is given by (129) with $A_{T}$ removed:

$$
A\left(B_{d}^{0} \rightarrow \phi K_{S}\right)=V_{c s} V_{c b}^{*}\left(P_{c}-P_{t}\right)+V_{u s} V_{u b}^{*}\left(P_{u}-P_{t}\right) .
$$

With

$$
\left|\frac{V_{u s} V_{u b}^{*}}{V_{c s} V_{c b}^{*}}\right| \leq 0.02, \quad \frac{P_{u}-P_{t}}{P_{c}-P_{t}}=\mathcal{O}(1)
$$

also in this decay single CKM phase dominates and as $\phi_{D}$ and $\phi_{M}$ are the same as in $B_{d}^{0} \rightarrow J / \psi K_{S}$ we find

$$
C_{\phi K_{S}}=0, \quad S_{\phi K_{S}}=S_{J / \psi K_{S}}=\sin 2 \beta .
$$

The equality of these two asymmetries need not be perfect as the $\phi$ meson is not entirely a $s \bar{s}$ state and the approximation of neglecting the second amplitude in (134) could be only true within a few percent. However, a detailed analysis shows [75] that these two asymmetries should be very close to each other within the SM: $\left|S_{\phi K_{S}}-S_{J / \psi K_{S}}\right| \leq 0.04$. Any strong violation of this bound would be a signal for new physics.

In view of this prediction, the first results on this asymmetry from BaBar [76] and Belle [77] are truely exciting:

$$
(\sin 2 \beta)_{\phi K_{S}}=\left\{\begin{array}{l}
-0.19 \pm 0.51 \text { (stat) } \pm 0.09 \text { (syst) (BaBar) } \\
-0.73 \pm 0.64 \text { (stat) } \pm 0.18 \text { (syst) (Belle). }
\end{array}\right.
$$

Consequently

$$
\begin{aligned}
& S_{\phi K_{s}}=-0.39 \pm 0.41, \quad C_{\phi K_{s}}=0.56 \pm 0.43, \\
& \left|S_{\phi K_{S}}-S_{J / \psi K_{S}}\right|=1.12 \pm 0.41
\end{aligned}
$$

where the result for $C_{\phi K_{S}}$, that is consistent with zero, comes solely from Belle. We observe that the bound $\left|S_{\phi K_{S}}-S_{J / \psi K_{S}}\right| \leq 0.04$ is violated by $2.7 \sigma$. While this is still insufficient to claim the presence of new physics, the fact that the two asymmetries are found to be quite different, invited a number of theorists to speculate what kind of new physics could be responsible for this difference. Some references are given in 78 . Enhanced QCD penguins, enhanced $Z^{0}$ penguins, rather involved supersymmetric scenarios have been suggested as possible origins of the departure from the SM prediction. I have no space to review these papers and although I find a few of them quite interesting, it is probably better to wait until the experimental errors decrease.

Of interest are also the measurements

$$
S_{\eta^{\prime} K_{S}}=\left\{\begin{array}{l}
0.02 \pm 0.35 \text { (BaBar) } \\
0.76 \pm 0.36 \text { (Belle). }
\end{array}\right.
$$


and $C_{\eta^{\prime} K_{S}}=-0.26 \pm 0.22$ from Belle that are fully consistent with $(\sin 2 \beta)_{J / \psi K_{S}}$. At first sight one could wonder why this asymmetry differs from $(\sin 2 \beta)_{\phi K_{S}}$ as the decay in question is also penguin dominated and $\eta^{\prime} \approx(s \bar{s})$, but the fact that $\eta^{\prime}$ deviates from a pure $(s \bar{s})$ state more than $\phi$ allows for some small contributions involving tree diagrams that could spoil the exact equality of these two asymmetries.

$B_{d}^{0} \rightarrow \pi^{+} \pi^{-}$and $\alpha$

This decay receives the contributions from both tree and penguin diagrams. The amplitude can be written as follows

$$
A\left(B_{d}^{0} \rightarrow \pi^{+} \pi^{-}\right)=V_{u d} V_{u b}^{*}\left(A_{T}+P_{u}\right)+V_{c d} V_{c b}^{*} P_{c}+V_{t d} V_{t b}^{*} P_{t}
$$

where

$$
V_{c d} V_{c b}^{*} \approx A \lambda^{3}, \quad V_{u d} V_{u b}^{*} \approx A \lambda^{3} R_{b} e^{i \gamma}, \quad V_{t d} V_{t b}^{*}=-V_{u d} V_{u b}^{*}-V_{c d} V_{c b}^{*} .
$$

Consequently

$$
A\left(B_{d}^{0} \rightarrow \pi^{+} \pi^{-}\right)=V_{u d} V_{u b}^{*}\left(A_{T}+P_{u}-P_{t}\right)+V_{c d} V_{c b}^{*}\left(P_{c}-P_{t}\right) .
$$

We next note that

$$
\left|\frac{V_{c d} V_{c b}^{*}}{V_{u d} V_{u b}^{*}}\right|=\frac{1}{R_{b}} \approx 2.5, \quad \frac{P_{c}-P_{t}}{A_{T}+P_{u}-P_{t}} \equiv \frac{P_{\pi \pi}}{T_{\pi \pi}} .
$$

Now the dominance of a single CKM amplitude in contrast to the cases considered until now is very uncertain and takes only place provided $P_{\pi \pi} \ll T_{\pi \pi}$. Let us assume first that this is indeed the case. Then this decay is dominated by a single CKM factor and a clean determination of the relevant CKM phase is possible. Indeed in this decay $\phi_{D}=\gamma$ and $\phi_{M}=-\beta$. Using (91) we find then $\left(\eta_{\pi \pi}=1\right)$

$$
a_{C P}^{\mathrm{int}}(\pi \pi)=\eta_{\pi \pi} \sin \left(2 \phi_{D}-2 \phi_{M}\right)=\sin 2(\gamma+\beta)=-\sin 2 \alpha
$$

and

$$
C_{\pi \pi}=0, \quad S_{\pi \pi}=\sin 2 \alpha .
$$

This should be compared with the first results from BaBar and Belle:

$$
\begin{aligned}
C_{\pi \pi} & =\left\{\begin{array}{l}
-0.30 \pm 0.25 \text { (stat) } \pm 0.04 \text { (syst) } \\
-0.77 \pm 0.27 \text { ( } \text { (stat) } \pm 0.08 \text { (syst) } \\
\text { (Belle) }
\end{array}\right. \\
S_{\pi \pi} & =\left\{\begin{array}{l}
-0.02 \pm 0.34 \text { (stat) } \pm 0.05 \text { (syst) (BaBar) } \\
-1.23 \pm 0.41 \text { (stat) } \pm 0.08 \text { (syst) (Belle). }
\end{array}\right.
\end{aligned}
$$

The results from BaBar are consistent with our expectations. Afterall $\alpha$ from the UT fit is in the ballpark of $90^{\circ}$. On the other hand Belle results indicate a non-zero asymmetry and a sizable contribution of the penguin diagrams invalidating our assumption $P_{\pi \pi} \ll T_{\pi \pi}$. Yet, as the results from BaBar and 
Belle are incompatible with each other, the present picture of this decay is not conclusive and one has to wait for better data.

The "QCD penguin pollution" discussed above has to be taken care of in order to extract $\alpha$. The well known strategy to deal with this "penguin problem" is the isospin analysis of Gronau and London [79. It requires however the measurement of $\operatorname{Br}\left(B^{0} \rightarrow \pi^{0} \pi^{0}\right)$ which is expected to be below $10^{-6}$ : a very difficult experimental task. For this reason several, rather involved, strategies have been proposed which avoid the use of $B_{d} \rightarrow \pi^{0} \pi^{0}$ in conjunction with $a_{C P}\left(\pi^{+} \pi^{-}, t\right)$. They are reviewed in $\left.99,13,14\right]$ 16]. The most recent analyses of $B \rightarrow \pi \pi$, also related to the determination of $\gamma$ and $(\bar{\varrho}, \bar{\eta})$, can be found in [3, 80, 81.

While I have some doubts that a precise value of $\alpha$ will follow in a foreseable future from this enterprise, one should also stress 82, 83, 44, that only a moderately precise measurement of $\sin 2 \alpha$ can be as useful for the UT as a precise measurement of the angle $\beta$. This is clear from table 1 that shows very large uncertainties in the indirect determination of $\sin 2 \alpha$.

\subsection{Decays to CP Non-Eigenstates}

\section{Preliminaries}

The strategies discussed below have the following general properties:

- $B_{d}^{0}\left(B_{s}^{0}\right)$ and their antiparticles $\bar{B}_{d}^{0}\left(\bar{B}_{s}^{0}\right)$ can decay to the same final state,

- Only tree diagrams contribute to the decay amplitudes,

- A full time dependent analysis of the four processes is required:

$$
B_{d, s}^{0}(t) \rightarrow f, \quad \bar{B}_{d, s}^{0}(t) \rightarrow f, \quad B_{d, s}^{0}(t) \rightarrow \bar{f}, \quad \bar{B}_{d, s}^{0}(t) \rightarrow \bar{f} .
$$

The latter analysis allows to measure

$$
\xi_{f}=\exp \left(i 2 \phi_{M}\right) \frac{A\left(\bar{B}^{0} \rightarrow f\right)}{A\left(B^{0} \rightarrow f\right)}, \quad \xi_{\bar{f}}=\exp \left(i 2 \phi_{M}\right) \frac{A\left(\bar{B}^{0} \rightarrow \bar{f}\right)}{A\left(B^{0} \rightarrow \bar{f}\right)} .
$$

It turns out then that

$$
\xi_{f} \cdot \xi_{\bar{f}}=F\left(\gamma, \phi_{M}\right)
$$

without any hadronic uncertainties, so that determining $\phi_{M}$ from other decays as discussed above, allows the determination of $\gamma$. Let us show this.

$$
B_{d}^{0} \rightarrow D^{ \pm} \pi^{\mp}, \bar{B}_{d}^{0} \rightarrow D^{ \pm} \pi^{\mp} \text { and } \gamma
$$

With $f=D^{+} \pi^{-}$the four decay amplitudes are given by

$$
\begin{aligned}
& A\left(B_{d}^{0} \rightarrow D^{+} \pi^{-}\right)=M_{f} A \lambda^{4} R_{b} e^{i \gamma}, \quad A\left(\bar{B}_{d}^{0} \rightarrow D^{+} \pi^{-}\right)=\bar{M}_{f} A \lambda^{2} \\
& A\left(\bar{B}_{d}^{0} \rightarrow D^{-} \pi^{+}\right)=\bar{M}_{\bar{f}} A \lambda^{4} R_{b} e^{-i \gamma}, \quad A\left(B_{d}^{0} \rightarrow D^{-} \pi^{+}\right)=M_{\bar{f}} A \lambda^{2}
\end{aligned}
$$


where we have factored out the CKM parameters, $A$ is a Wolfenstein paramater and $M_{i}$ stand for the rest of the amplitudes that generally are subject to large hadronic uncertainties. The important point is that each of these transitions receives the contribution from a single phase so that

$$
\xi_{f}^{(d)}=e^{-i(2 \beta+\gamma)} \frac{1}{\lambda^{2} R_{b}} \frac{\bar{M}_{f}}{M_{f}}, \quad \xi_{\bar{f}}^{(d)}=e^{-i(2 \beta+\gamma)} \lambda^{2} R_{b} \frac{\bar{M}_{\bar{f}}}{M_{\bar{f}}} .
$$

Now, as CP is conserved in QCD we simply have

$$
M_{f}=\bar{M}_{\bar{f}}, \quad \bar{M}_{f}=M_{\bar{f}}
$$

and consequently 84

$$
\xi_{f}^{(d)} \cdot \xi_{\bar{f}}^{(d)}=e^{-i 2(2 \beta+\gamma)}
$$

as promised. The phase $\beta$ is already known with high precision and consequently $\gamma$ can be determined. Unfortunately as seen in (148) and (149), the relevant interefences are $\mathcal{O}\left(\lambda^{2}\right)$ and the execution of this strategy is a very difficult experimental task. See [85] for an interesting discussion.

$B_{s}^{0} \rightarrow D_{s}^{ \pm} K^{\mp}, \bar{B}_{s}^{0} \rightarrow D_{s}^{ \pm} K^{\mp}$ and $\gamma$

Replacing the d-quark by the s-quark in the strategy just discussed allows to solve the latter problem. With $f=D_{s}^{+} K^{-}$equations (148) and (149) are replaced by

$$
\begin{aligned}
& A\left(B_{s}^{0} \rightarrow D_{s}^{+} K^{-}\right)=M_{f} A \lambda^{3} R_{b} e^{i \gamma}, \quad A\left(\bar{B}_{s}^{0} \rightarrow D_{s}^{+} K^{-}\right)=\bar{M}_{f} A \lambda^{3} \\
& A\left(\bar{B}_{s}^{0} \rightarrow D_{s}^{-} K^{+}\right)=\bar{M}_{\bar{f}} A \lambda^{3} R_{b} e^{-i \gamma},
\end{aligned}
$$

Proceeding as in the previous strategy one finds 86

$$
\xi_{f}^{(s)} \cdot \xi_{\bar{f}}^{(s)}=e^{-i 2\left(2 \beta_{s}+\gamma\right)}
$$

with $\xi_{f}^{(s)}$ and $\xi_{\bar{f}}^{(s)}$ being the analogs of $\xi_{f}^{(d)}$ and $\xi_{\bar{f}}^{(d)}$, respectively. Now, all interferring amplitudes are of a similar size. With $\beta_{s}$ extracted one day from the asymmetry in $B_{s}^{0}\left(\bar{B}_{s}^{0}\right) \rightarrow J / \psi \phi$, the angle $\gamma$ can be determined.

$B^{ \pm} \rightarrow D^{0} K^{ \pm}, B^{ \pm} \rightarrow \bar{D}^{0} K^{ \pm}$and $\gamma$

By replacing the spectator s-quark in the last strategy through the u-quark one arrives at decays of $B^{ \pm}$that can be used to extract $\gamma$. Also this strategy is unaffected by penguin contributions. Moreover, as particle-antiparticle mixing is absent here, $\gamma$ can be measured directly without any need for phases in the mixing. Both these features make it plausible that this strategy, not involving to first approximation any loop diagrams, is particularly suited for the determination of $\gamma$ without any new physics pollution. 
By considering six decay rates $B^{ \pm} \rightarrow D_{C P}^{0} K^{ \pm}, B^{+} \rightarrow D^{0} K^{+}, \bar{D}^{0} K^{+}$and $B^{-} \rightarrow D^{0} K^{-}, \bar{D}^{0} K^{-}$where $\left.D_{C P}^{0}=\left(D^{0}+\bar{D}^{0}\right) / \sqrt{2}\right)$ is a CP eigenstate, and noting that

$$
\begin{aligned}
& A\left(B^{+} \rightarrow \bar{D}^{0} K^{+}\right)=A\left(B^{-} \rightarrow D^{0} K^{-}\right), \\
& A\left(B^{+} \rightarrow D^{0} K^{+}\right)=A\left(B^{-} \rightarrow \bar{D}^{0} K^{-}\right) e^{2 i \gamma}
\end{aligned}
$$

the well known triangle construction due to Gronau and Wyler 87 allows to determine $\gamma$. However, the method is not without problems. The detection of $D_{C P}^{0}$, that is necessary for this determination because $K^{+} \bar{D}^{0} \neq K^{+} D^{0}$, is experimentally challenging. Moreover, the small branching ratios of the colour supressed channels in (157) and the absence of this suppression in the two remaining channels in (156) imply a rather squashed triangle thereby making the extraction of $\gamma$ very difficult. Variants of this method that could be more promising are discussed in [88, 89].

\section{Other Clean Strategies for $\gamma$ and $\beta$}

The three strategies discussed above can be generalized to other decays. In particular 88, 90.

- $2 \beta+\gamma$ and $\gamma$ can be measured in

$$
B_{d}^{0} \rightarrow K_{S} D^{0}, K_{S} \bar{D}^{0}, \quad B_{d}^{0} \rightarrow \pi^{0} D^{0}, \pi^{0} \bar{D}^{0}
$$

and the corresponding $\mathrm{CP}$ conjugated channels,

- $2 \beta_{s}+\gamma$ and $\gamma$ can be measured in

$$
B_{s}^{0} \rightarrow \phi D^{0}, \phi \bar{D}^{0}, \quad B_{s}^{0} \rightarrow K_{S}^{0} D^{0}, K_{S} \bar{D}^{0}
$$

and the corresponding $\mathrm{CP}$ conjugated channels,

- $\gamma$ can be measured by generalizing the Gronau-Wyler construction to $B^{ \pm} \rightarrow D^{0} \pi^{ \pm}, \bar{D}^{0} \pi^{ \pm}$and to $B_{c}$ decays [1]:

$$
B_{c}^{ \pm} \rightarrow D^{0} D_{s}^{ \pm}, \bar{D}^{0} D_{s}^{ \pm}, \quad B_{c}^{ \pm} \rightarrow D^{0} D^{ \pm}, \bar{D}^{0} D^{ \pm} .
$$

In this context I can strongly recommend recent papers by Fleischer 90 that while discussing these decays go far beyond the methods presented here. It appears that the methods discussed in this subsection may give useful results at later stages of CP-B investigations, in particular at LHC-B and BTeV.

\subsection{U-Spin Strategies}

\section{Preliminaries}

Useful strategies for $\gamma$ using the U-spin symmetry have been proposed in 92, 93. The first strategy involves the decays $B_{d, s}^{0} \rightarrow \psi K_{S}$ and $B_{d, s}^{0} \rightarrow$ $D_{d, s}^{+} D_{d, s}^{-}$. The second strategy involves $B_{s}^{0} \rightarrow K^{+} K^{-}$and $B_{d}^{0} \rightarrow \pi^{+} \pi^{-}$. They 
are unaffected by FSI and are only limited by U-spin breaking effects. They are promising for Run II at FNAL and in particular for LHC-B.

A method of determining $\gamma$, using $B^{+} \rightarrow K^{0} \pi^{+}$and the U-spin related processes $B_{d}^{0} \rightarrow K^{+} \pi^{-}$and $B_{s}^{0} \rightarrow \pi^{+} K^{-}$, was presented in 94. A general discussion of U-spin symmetry in charmless B decays and more references to this topic can be found in [16, 95. I will only briefly discuss the method in 93.

$B_{d}^{0} \rightarrow \pi^{+} \pi^{-}, B_{s}^{0} \rightarrow K^{+} K^{-}$and $(\gamma, \beta)$

Replacing in $B_{d}^{0} \rightarrow \pi^{+} \pi^{-}$the $d$ quark by the $s$ quark we obtain the decay $B_{s}^{0} \rightarrow K^{+} K^{-}$. The amplitude can be then written in analogy to (141) as follows

$$
A\left(B_{s}^{0} \rightarrow K^{+} K^{-}\right)=V_{u s} V_{u b}^{*}\left(A_{T}^{\prime}+P_{u}^{\prime}-P_{t}^{\prime}\right)+V_{c s} V_{c b}^{*}\left(P_{c}^{\prime}-P_{t}^{\prime}\right) .
$$

This formula differs from (141) only by $d \rightarrow s$ and the primes on the hadronic matrix elements that in principle are different in these two decays. As

$$
V_{c s} V_{c b}^{*} \approx A \lambda^{2}, \quad V_{u s} V_{u b}^{*} \approx A \lambda^{4} R_{b} e^{i \gamma},
$$

the second term in (161) is even more important than the corresponding term in the case of $B_{d}^{0} \rightarrow \pi^{+} \pi^{-}$. Consequently $B_{d}^{0} \rightarrow K^{+} K^{-}$taken alone does not offer a useful method for the determination of the CKM phases. On the other hand, with the help of the U-spin symmetry of strong interations, it allows roughly speaking to determine the penguin contributions in $B_{d}^{0} \rightarrow \pi^{+} \pi^{-}$and consequently the extraction of $\beta$ and $\gamma$.

Indeed, from the U-spin symmetry we have

$$
\frac{P_{\pi \pi}}{T_{\pi \pi}}=\frac{P_{c}-P_{t}}{A_{T}+P_{u}-P_{t}}=\frac{P_{c}^{\prime}-P_{t}^{\prime}}{A_{T}^{\prime}+P_{u}^{\prime}-P_{t}^{\prime}}=\frac{P_{K K}}{T_{K K}} \equiv d e^{i \delta}
$$

where $d$ is a real non-perturbative parameter and $\delta$ a strong phase. Measuring $S_{f}$ and $C_{f}$ for both decays and extracting $\beta_{s}$ from $B_{s}^{0} \rightarrow J / \psi \phi$, we can determine four unknows: $d, \delta, \beta$ and $\gamma$ subject mainly to U-spin breaking corrections. A recent analysis using these ideas can be found in [81.

\subsection{Constraints for $\gamma$ from $B \rightarrow \pi K$}

\section{Preliminaries}

The recent developments involve also the extraction of the angle $\gamma$ from the decays $B \rightarrow \pi K$. The modes $B^{ \pm} \rightarrow \pi^{\mp} K^{0}, B^{ \pm} \rightarrow \pi^{0} K^{ \pm}, B_{d}^{0} \rightarrow \pi^{\mp} K^{ \pm}$and $B_{d}^{0} \rightarrow \pi^{0} K^{0}$ have been observed by the CLEO, BaBar and Belle collaborations and should allow us to obtain direct information on $\gamma$ when the errors on branching ratios and the $\mathrm{CP}$ asymmetries decrease. The latter are still consistent with zero. The progress on the accuracy of these measurements is 
slow but steady and they will certainly give an interesting insight into the flavour dynamics and QCD dynamics one day.

There has been a large theoretical activity in this field during the last six years. The main issues here are the final state interactions (FSI), SU(3) symmetry breaking effects and the importance of electroweak penguin contributions. Several interesting ideas have been put forward to extract the angle $\gamma$ in spite of large hadronic uncertainties in $B \rightarrow \pi K$ decays 96, 97, 98, 99 100, 101.

Three strategies for bounding and determining $\gamma$ have been proposed. The "mixed" strategy [96] uses $B_{d}^{0} \rightarrow \pi^{0} K^{ \pm}$and $B^{ \pm} \rightarrow \pi^{ \pm} K$. The "charged" strategy [101] involves $B^{ \pm} \rightarrow \pi^{0} K^{ \pm}, \pi^{ \pm} K$ and the "neutral" strategy 99] the modes $B_{d}^{0} \rightarrow \pi^{\mp} K^{ \pm}, \pi^{0} K^{0}$. General parametrizations for the study of the FSI, SU(3) symmetry breaking effects and of the electroweak penguin contributions in these channels have been presented in [98, 99, 100. Moreover, general parametrizations by means of Wick contractions 102, 103 have been proposed. They can be used for all two-body B-decays. These parametrizations should turn out to be useful when the data improve.

Parallel to these efforts an important progress has been made by developing approaches for the calculation of the hadronic matrix elements of local operators in QCD beyond the standard factorization method. These are in particular the QCD factorization approach [104, the perturbative QCD approach [105] and the soft-collinear effective theory [106. Moreover new methods to calculate exclusive hadronic matrix elements from QCD light-cone sum rules have been developed in [107. While, in my opinion, an important progress in evaluating non-leptonic amplitudes has been made in these papers, the usefulness of this recent progress at the quantitative level has still to be demonstrated when the data improve.

\section{A General Parametrization for $B \rightarrow \pi K$}

In order to illustrate the complexity of the extraction of $\gamma$ from these decays let me describe briefly the general parametrization for the mixed, charged and neutral strategies, developed in 1998 in collaboration with Robert Fleischer 99.

The isospin symmetry implies in each case one relation between the relevant amplitudes:

$$
\begin{aligned}
& A\left(B^{+} \rightarrow \pi^{+} K^{0}\right)+A\left(B_{d}^{0} \rightarrow \pi^{-} K^{+}\right)=-\left[T+P_{\mathrm{EW}}^{\mathrm{C}}\right] \\
& A\left(B^{+} \rightarrow \pi^{+} K^{0}\right)+\sqrt{2} A\left(B^{+} \rightarrow \pi^{0} K^{+}\right)=-\left[(T+C)+P_{\mathrm{EW}}\right]=3 A_{3 / 2}(165) \\
& \sqrt{2} A\left(B_{d}^{0} \rightarrow \pi^{0} K^{0}\right)+A\left(B_{d}^{0} \rightarrow \pi^{-} K^{+}\right)=-\left[(T+C)+P_{\mathrm{EW}}\right]=3 A_{3 / 2}(166
\end{aligned}
$$

where $T$ stands for tree, $C$ for colour suppressed tree, $P_{\mathrm{EW}}$ for electroweak penguins and $P_{\mathrm{EW}}^{\mathrm{C}}$ for colour suppressed electroweak penguins. $A_{3 / 2}$ is an isospin amplitude. In particular we have 


$$
T+C=|T+C| e^{i \delta_{T+C}} e^{i \gamma}, \quad P_{\mathrm{EW}}=-\left|P_{\mathrm{EW}}\right| e^{i \delta_{\mathrm{EW}}}
$$

where the $\delta_{i}$ denote the strong interaction phases.

The QCD penguins, absent in (164)-(166), enter the analysis in the following manner:

$$
\begin{aligned}
& P_{c h} \equiv A\left(B^{+} \rightarrow \pi^{+} K^{0}\right)=-\left(1-\frac{\lambda^{2}}{2}\right) \lambda^{2} A\left[1+\varrho_{c h} e^{i \theta_{c h}} e^{i \gamma}\right]\left|P_{t c}^{c h}\right| e^{i \delta_{t c}^{c h}} \\
& P_{n} \equiv \sqrt{2} A\left(B_{d}^{0} \rightarrow \pi^{0} K^{0}\right)=-\left(1-\frac{\lambda^{2}}{2}\right) \lambda^{2} A\left[1+\varrho_{n} e^{i \theta_{n}} e^{i \gamma}\right]\left|P_{t c}^{n}\right| e^{i \delta_{t c}^{n}}
\end{aligned}
$$

where the terms proportional to $\varrho_{c h, n}$ parametrize $u$-penguin and rescattering effects and the last factors stand for the difference of $t$ and $c$ penguins.

The relevant parameters in the three strategies in question are

$$
\begin{aligned}
& r=\frac{|T|}{\sqrt{\left|P_{c h}\right|^{2}}}, \quad q=\left|\frac{P_{\mathrm{EW}}^{\mathrm{C}}}{T}\right| e^{i \bar{\omega}}, \quad \delta=\delta_{T}-\delta_{t c} \\
& r_{c h}=\frac{|T+C|}{\sqrt{\left|P_{c h}\right|^{2}}}, \quad q_{c h}=\left|\frac{P_{\mathrm{EW}}}{T+C}\right| e^{i \omega}, \quad \delta_{c h}=\delta_{T+C}-\delta_{t c}^{c h} \\
& r_{n}=\frac{|T+C|}{\sqrt{\left|P_{n}\right|^{2}}}, \quad q_{n}=q_{c h}, \quad \delta_{n}=\delta_{T+C}-\delta_{t c}^{n} .
\end{aligned}
$$

The virtue of this general parametrization, is the universality of various formulae for quantities of interest, not shown here due to the lack of space. In order to study a given strategy, the relevant parameters listed above have to be inserted in these formulae.

The formulae given above are not sufficiently informative for a determination of $\gamma$. To proceed further one has to use $S U(3)$ flavour symmetry. This allows to fix $r_{c h}, r_{n}$ and $q_{c h}=q_{n}$. On the other hand $r$ and $q$ are not determined by $S U(3)$ and their values have to be estimated by some dynamical assumptions like factorization. Consequently the mixed strategy has larger theoretical uncertainties than the other two strategies.

We have then, respectively, 101, 108.

$$
\begin{aligned}
& q_{c h}=q_{n}=0.70\left[\frac{0.37}{R_{b}}\right]=0.70 \pm 0.08, \\
& r_{c h}=\sqrt{2}\left|\frac{V_{u s}}{V_{u d}}\right| \frac{F_{K}}{F_{\pi}} \sqrt{\frac{B r\left(B^{ \pm} \rightarrow \pi^{ \pm} \pi^{0}\right)}{B r\left(B^{ \pm} \rightarrow \pi^{ \pm} K^{0}\right)}}=0.20 \pm 0.02
\end{aligned}
$$

with $|T+C|$ in $r_{c h}$ extracted from $B^{ \pm} \rightarrow \pi^{ \pm} \pi^{0}$. The last number is my own estimate. Similarly one finds 99

$$
r_{n}=\left|\frac{V_{u s}}{V_{u d}}\right| \frac{F_{K}}{F_{\pi}} \sqrt{\frac{B r\left(B^{ \pm} \rightarrow \pi^{ \pm} \pi^{0}\right)}{B r\left(B_{d}^{0} \rightarrow \pi^{0} K^{0}\right)}} \sqrt{\frac{\tau\left(B_{d}^{0}\right)}{\tau\left(B^{ \pm}\right)}}=0.17 \pm 0.02 .
$$


As demonstrated in a vast number of papers [96, 98, 99, 100, 101, these strategies imply interesting bounds on $\gamma$ that not necessarily agree with the values extracted from the UT analysis of section 3. In particular already in 1999 combining the neutral and charged strategies 99. we have found that the 1999 data on $B \rightarrow \pi K$ favour $\gamma$ in the second quadrant, which is in conflict with the standard analysis of the unitarity triangle that implied $\gamma=(62 \pm 7)^{\circ}$. Other arguments for $\cos \gamma<0$ using $B \rightarrow P P, P V$ and $V V$ decays were given also in [109. Recent analyses of $B \rightarrow \pi K$ by various authors find also that $\gamma>90^{\circ}$ is favoured by the $B \rightarrow \pi K$ data. Most recent reviews can be found in 3 . See also [110.

In view of sizable theoretical uncertainties in the analyses of $B \rightarrow \pi K$ and of still significant experimental errors in the corresponding branching ratios it is not yet clear whether the discrepancy in question is serious. For instance 111] sizable contributions of the so-called charming penguins to the $B \rightarrow \pi K$ amplitudes could shift $\gamma$ extracted from these decays below $90^{\circ}$ but at present these contributions cannot be calculated reliably. Similar role could be played by annihilation contributions [105] and large non-factorizable SU(3) breaking effects 99. Also, new physics contributions in the electroweak penguin sector could shift $\gamma$ to the first quadrant [99]. It should be however emphasized that the problem with the angle $\gamma$, if it persisted, would put into difficulties not only the SM but also the full class of MFV models in which the lower bound on $\Delta M_{s} / \Delta M_{d}$ implies $\gamma<90^{\circ}$. In any case it will be exciting to follow the developments in this field.

\section{$6 K^{+} \rightarrow \pi^{+} \nu \bar{\nu}$ and $K_{\mathrm{L}} \rightarrow \pi^{0} \nu \bar{\nu}$}

The rare decays $K^{+} \rightarrow \pi^{+} \nu \bar{\nu}$ and $K_{\mathrm{L}} \rightarrow \pi^{0} \nu \bar{\nu}$ are very promising probes of flavour physics within the SM and possible extensions, since they are governed by short distance interactions. They proceed through $Z^{0}$-penguin and box diagrams. As the required hadronic matrix elements can be extracted from the leading semileptonic decays and other long distance contributions turn out to be negligible 112, the relevant branching ratios can be computed to an exceptionally high degree of precision [113, 114, 115. The main theoretical uncertainty in the $\mathrm{CP}$ conserving decay $K^{+} \rightarrow \pi^{+} \nu \bar{\nu}$ originates in the value of $m_{\mathrm{c}}\left(\mu_{c}\right)$. It has been reduced through NLO corrections down to $\pm 7 \%$ 113, 114 at the level of the branching ratio. The dominantly CP-violating decay $K_{\mathrm{L}} \rightarrow$ $\pi^{0} \nu \bar{\nu}$ [116] is even cleaner as only the internal top contributions matter. The theoretical error for $\operatorname{Br}\left(K_{\mathrm{L}} \rightarrow \pi^{0} \nu \bar{\nu}\right)$ amounts to $\pm 2 \%$ and is safely negligible.

\subsection{Branching Ratios}

The basic formulae for the branching ratios are given as follows

$$
\operatorname{Br}\left(K^{+} \rightarrow \pi^{+} \nu \bar{\nu}\right)=\kappa_{+} \cdot\left[\left(\operatorname{Im} F_{t}\right)^{2}+\left(\operatorname{Re} F_{c}+\operatorname{Re} F_{t}\right)^{2}\right],
$$




$$
\operatorname{Br}\left(K_{\mathrm{L}} \rightarrow \pi^{0} \nu \bar{\nu}\right)=\kappa_{\mathrm{L}} \cdot\left(\operatorname{Im} F_{t}\right)^{2}
$$

where

$$
F_{c}=\frac{\lambda_{c}}{\lambda} P_{0}(X), \quad F_{t}=\frac{\lambda_{t}}{\lambda^{5}} X\left(x_{t}\right) .
$$

Here $\lambda_{i}=V_{i s}^{*} V_{i d}$ and

$$
\kappa_{+}=4.75 \cdot 10^{-11}, \quad \kappa_{\mathrm{L}}=2.08 \cdot 10^{-10}
$$

include isospin breaking corrections in relating $K^{+} \rightarrow \pi^{+} \nu \bar{\nu}$ and $K_{\mathrm{L}} \rightarrow \pi^{0} \nu \bar{\nu}$ to $K^{+} \rightarrow \pi^{0} e^{+} \nu$, respectively [117. Next

$$
X\left(x_{t}\right)=1.52 \cdot\left[\frac{\bar{m}_{\mathrm{t}}\left(m_{\mathrm{t}}\right)}{167 \mathrm{GeV}}\right]^{1.15}
$$

represents internal top contribution and $P_{0}(X)=0.39 \pm 0.06$ results from the internal charm contribution [113. The numerical values in (179) and for $P_{0}(X)$ differ from [114 due to a different value of $\lambda=0.224$ used here.

Imposing all existing constraints on the CKM matrix one finds [118]

$$
\begin{aligned}
& \operatorname{Br}\left(K^{+} \rightarrow \pi^{+} \nu \bar{\nu}\right)=(7.7 \pm 1.1) \cdot 10^{-11}, \\
& \operatorname{Br}\left(K_{\mathrm{L}} \rightarrow \pi^{0} \nu \bar{\nu}\right)=(2.6 \pm 0.5) \cdot 10^{-11}
\end{aligned}
$$

where the errors come dominantly from the uncertainties in the CKM parameters. Similar results are found in 119 . The first result should be compared with the measurements of AGS E787 collaboration at Brookhaven [120] that observing two events for this very rare decay finds

$$
\operatorname{Br}\left(K^{+} \rightarrow \pi^{+} \nu \bar{\nu}\right)=\left(15.7_{-8.2}^{+17.5}\right) \cdot 10^{-11} .
$$

This is a factor of 2 above the SM expectation. Even if the errors are substantial and the result is compatible with the SM, the branching ratio (183) implies already a non-trivial lower bound on $\left|V_{t d}\right|$ [120, 121].

The present upper bound on $\operatorname{Br}\left(K_{\mathrm{L}} \rightarrow \pi^{0} \nu \bar{\nu}\right)$ from the $\mathrm{KTeV}$ experiment at Fermilab 122 reads

$$
\operatorname{Br}\left(K_{\mathrm{L}} \rightarrow \pi^{0} \nu \bar{\nu}\right)<5.9 \cdot 10^{-7} .
$$

This is about four orders of magnitude above the SM expectation (182). Moreover this bound is substantially weaker than the model independent bound [123] from isospin symmetry:

$$
\operatorname{Br}\left(K_{\mathrm{L}} \rightarrow \pi^{0} \nu \bar{\nu}\right)<4.4 \cdot \operatorname{Br}\left(K^{+} \rightarrow \pi^{+} \nu \bar{\nu}\right)
$$

which through (183) gives

$$
\operatorname{Br}\left(K_{\mathrm{L}} \rightarrow \pi^{0} \nu \bar{\nu}\right)<1.6 \cdot 10^{-9}(90 \% \text { C.L. })
$$




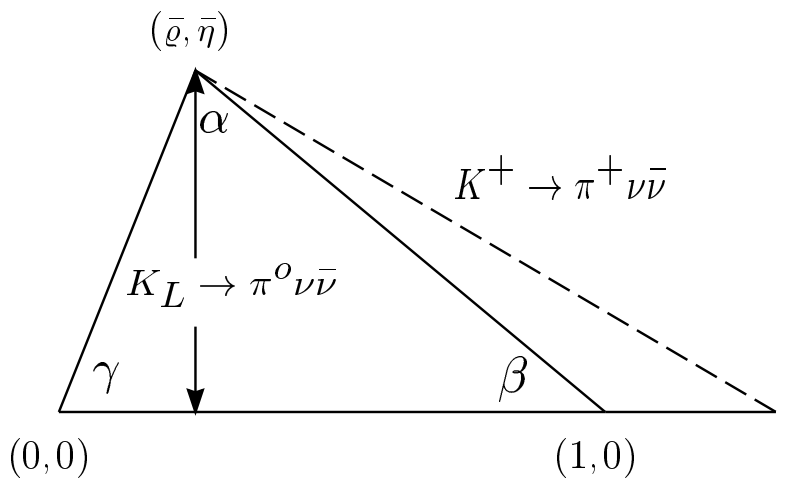

Fig. 9. Unitarity triangle from $K \rightarrow \pi \nu \bar{\nu}$.

\subsection{Unitarity Triangle and $\sin 2 \beta$ from $K \rightarrow \pi \nu \bar{\nu}$}

The measurement of $\operatorname{Br}\left(K^{+} \rightarrow \pi^{+} \nu \bar{\nu}\right)$ and $\operatorname{Br}\left(K_{\mathrm{L}} \rightarrow \pi^{0} \nu \bar{\nu}\right)$ can determine the unitarity triangle completely, (see fig. 9) [82. The explicit formulae can be found in $21,6,82$. The most interesting in this context are very clean determinations of $\sin 2 \beta$ and $\operatorname{Im} \lambda_{t}$ that are free not only from hadronic uncertainties but also parametric uncertainties like $\left|V_{c b}\right|$ and $m_{c}$. The determination of $\left|V_{t d}\right|$ is also theoretically clean but its precision depends on the accuracy with which $\left|V_{c b}\right|$ and $m_{c}$ are known. Also the scale uncertainties in $\left|V_{t d}\right|$ amount to $4 \%$ at the NLO [113. They should be significantly reduced through a calculation of NNLO corrections to the charm contribution that is in progress and should be available in 2004 .

Assuming that the branching ratios will be known to within $\pm 10 \%$ we expect the following accuracy in this decade

$$
\sigma(\sin 2 \beta)= \pm 0.04, \quad \sigma\left(\operatorname{Im} \lambda_{t}\right)= \pm 5 \%, \quad \sigma\left(\left|V_{t d}\right|\right)= \pm 7 \% .
$$

The comparison with the corresponding determinations in B decays will offer a very good test of flavour dynamics and $\mathrm{CP}$ violation in the SM and a powerful tool to probe the physics beyond it.

\subsection{Concluding Remarks}

As the theorists were able to calculate the branching ratios for these decays rather precisely, the future of this field is in the hands of experimentalists and depends on the financial support that is badly needed. The experimental outlook for these decays has been reviewed in [124, 125]. The future of $K^{+} \rightarrow$ $\pi^{+} \nu \bar{\nu}$ depends on the AGS E949 and the CKM experiment at Fermilab. In the case of $K_{\mathrm{L}} \rightarrow \pi^{0} \nu \bar{\nu}$ these are the KEK E391a experiment, KOPIO at Brookhaven (BNL E926) and an experiment at the $50 \mathrm{GeV}$ JHF in Japan 
that should be able to collect 1000 events at the end of this decade. Both KOPIO and JHF should provide very important measurements of this goldplated decay. For a recent theoretical review see [118].

\section{Minimal Flavour Violation Models}

\subsection{Preliminaries}

We have defined this class of models in Section 1. Here I would like just to list four interesting properties of these models that are independent of particular parameters present in these models. Other relations can be found in [126]. These are:

- There exists a universal unitarity triangle (UUT) 24] common to all these models and the SM that can be constructed by using measurable quantities that depend on the CKM parameters but are not polluted by the new parameters present in the extensions of the SM. The UUT can be constructed, for instance, by using $\sin 2 \beta$ from $a_{\psi K_{S}}$ and the ratio $\Delta M_{s} / \Delta M_{d}$. The relevant formulae can be found in Section 3 and in 24, 127, where also other quantities suitable for the determination of the UUT are discussed.

- $\quad(\sin 2 \beta)_{J / \psi K_{S}}=(\sin 2 \beta)_{\phi K_{S}}=(\sin 2 \beta)_{\pi \nu \bar{\nu}}$

- For given $a_{\psi K_{\mathrm{S}}}$ and $\operatorname{Br}\left(K^{+} \rightarrow \pi^{+} \nu \bar{\nu}\right)$ only two values of $\operatorname{Br}\left(K_{\mathrm{L}} \rightarrow\right.$ $\left.\pi^{0} \nu \bar{\nu}\right)$ are possible in the full class of MFV models, independently of any new parameters present in these models [127. Consequently, measuring $\operatorname{Br}\left(K_{\mathrm{L}} \rightarrow \pi^{0} \nu \bar{\nu}\right)$ will either select one of these two possible values or rule out all MFV models. The present experimental bound on $\operatorname{Br}\left(K^{+} \rightarrow \pi^{+} \nu \bar{\nu}\right)$ and $\sin 2 \beta \leq 0.80$ imply an absolute upper bound $\operatorname{Br}\left(K_{\mathrm{L}} \rightarrow \pi^{0} \nu \bar{\nu}\right)<4.9 \cdot 10^{-10}$ (90\% C.L.) [127] in the MFV models that is stronger than the bound in (186).

- There exists a correlation between $\operatorname{Br}\left(B_{d, s} \rightarrow \mu \bar{\mu}\right)$ and $\Delta M_{d, s}$ [128]:

$$
\frac{B r\left(B_{s} \rightarrow \mu \bar{\mu}\right)}{B r\left(B_{d} \rightarrow \mu \bar{\mu}\right)}=\frac{\hat{B}_{d}}{\hat{B}_{s}} \frac{\tau\left(B_{s}\right)}{\tau\left(B_{d}\right)} \frac{\Delta M_{s}}{\Delta M_{d}}
$$

that is practically free of theoretical uncertainties as $\hat{B}_{s} / \hat{B}_{d}=1$ up to small breaking $S U(3)$ breaking corrections. Similar correlations between $\operatorname{Br}\left(B_{d, s} \rightarrow \mu \bar{\mu}\right)$ and $\Delta M_{d, s}$, respectively, allow rather precise predictions for $\operatorname{Br}\left(B_{d, s} \rightarrow \mu \bar{\mu}\right)$ within the MFV models once $\Delta M_{d, s}$ are known 128.

\subsection{Universal Unitarity Triangle}

The presently available quantities that do not depend on the new physics parameters within the MFV models and therefore can be used to determine the UUT are $R_{t}$ from $\Delta M_{d} / \Delta M_{s}$ by means of (104), $R_{b}$ from $\left|V_{u b} / V_{c b}\right|$ by means of (12) and $\sin 2 \beta$ extracted from the CP asymmetry in $B_{d}^{0} \rightarrow \psi K_{S}$. 
Using only these three quantities, we show in figure [ 6 the allowed universal region for $(\bar{\varrho}, \bar{\eta})$ (the larger ellipse) in the MFV models as obtained recently in an update of 44]. The results for various quantities of interest related to this UUT are collected in table 1 Similar analysis has been done in 26.

It should be stressed that any MFV model that is inconsistent with the broader allowed region in figure [ and the UUT column in table 1 is ruled out. We observe that there is little room for MFV models that in their predictions for UT differ significantly from the SM. It is also clear that to distinguish the SM from the MFV models on the basis of the analysis of the UT of Section 3 , will require considerable reduction of theoretical uncertainties.

\subsection{Models with Universal Extra Dimensions}

In view of the difficulty in distinguishing various MFV models on the basis of the standard analysis of UT from each other, it is essential to study other FCNC processes as rare $\mathrm{B}$ and $\mathrm{K}$ decays and radiative $\mathrm{B}$ decays like $B \rightarrow X_{s} \gamma$ and $B \rightarrow X_{s} \mu^{+} \mu^{-}$. In the case of MSSM at low $\tan \beta$ such an analyses can be found in [50, 129]. Recently a very extensive analysis of all relevant FCNC processes in a SM with one universal extra dimension [130] has been presented in 131, 132. In this model all standard model fields can propagate in the fifth dimension and the FCNC processes are affected by the exchange of the Kaluza-Klein particles in loop diagrams. The most interesting results of 131] 132, 133] are the enhancements of $\operatorname{Br}\left(K^{+} \rightarrow \pi^{+} \nu \bar{\nu}\right)$ and $\operatorname{Br}(B \rightarrow$ $\left.X_{s} \mu^{+} \mu^{-}\right)$, strong suppressions of $\operatorname{Br}\left(B \rightarrow X_{s} \gamma\right)$ and $\operatorname{Br}\left(B \rightarrow X_{s}\right.$ gluon $)$ and a significant downward shift of the zero $\hat{s}_{0}$ in the forward-backward asymmetry in $\operatorname{Br}\left(B \rightarrow X_{s} \mu^{+} \mu^{-}\right)$.

\section{Outlook}

Let me finish these lectures with my personal expectations for the coming years with regard to the CKM matrix and FCNC processes.

\subsection{Phase 1 (2003-2007)}

In this phase the determination of the CKM matrix will be governed by

$$
V_{u s}, \quad\left|V_{c b}\right|, \quad a_{\psi K_{S}}, \quad \Delta M_{d} / \Delta M_{s} .
$$

These four quantities are sufficient to determine the full CKM matrix and suggest a new set of fundamental variables 44]

$$
V_{u s}, \quad\left|V_{c b}\right|, \quad \beta, \quad R_{t} .
$$

The precision of this determination will depend on the accuracy with which $a_{\psi K_{S}}$ and $\Delta M_{d} / \Delta M_{s}$ will be measured and the non-perturbative ratio $\xi$ calculated by lattice and QCD sum rules methods. 
An important role will also be played by

$\varepsilon_{K}, \quad\left|V_{u b} / V_{c b}\right|$

but this will depend on the reduction of the hadronic uncertainties in $\hat{B}_{K}$ and in the determination of $\left|V_{u b}\right| \underline{3}$. Note that $\varepsilon_{K}$ and $\left|V_{u b} / V_{c b}\right|$ combined with (190) can tell us whether the CP violation in the $\mathrm{K}$-system is consistent with the one observed in the B-system.

Very important is the clarification of the possible discrepancy between the measurements of the angle $\beta$ by means of $a_{J / \psi K_{S}}$ and $a_{\phi K_{S}}$ that if confirmed would imply new sources of CP violation. Similarly the status of CP violation in $B_{d}^{0} \rightarrow \pi^{+} \pi^{-}$and in $B \rightarrow \pi K$ decays should be clarified in this phase but this will depend on the theoretical progress in non-perturbative methods. We should also be able to get some information about $\gamma$ not only from $B \rightarrow \pi K$ at $\mathrm{B}$ factories but also by means of U-spin strategies in conjuction with the data from Run II at Tevatron.

During this phase we should also have new data on $\operatorname{Br}\left(K^{+} \rightarrow \pi^{+} \nu \bar{\nu}\right)$ from AGS E949 and first data from the CKM experiment at Fermilab. The comparison of these data with the implications of $\Delta M_{d} / \Delta M_{s}$ should be very interesting 114, 121, 131, 81. It would be particularly exciting if the central value did not decrease below the one in (183), that is roughly by a factor of two higher than the SM value. In any case these data should have a considerable impact on $\left|V_{t d}\right|$ and the unitarity triangle.

We will also have new data on $B_{s} \rightarrow \mu^{+} \mu^{-}, B \rightarrow X_{s} \nu \bar{\nu}, B \rightarrow X_{s} \gamma$, $B \rightarrow X_{s} \mu \bar{\mu}$ as well as on related exclusive channels. All these decays are governed by the CKM element $\left|V_{t s}\right|$ that is already well determined by the unitarity of the CKM matrix $\left|V_{t s}\right| \approx\left|V_{c b}\right|$. Consequently I do not expect that these decays will play an important role in the CKM fits. On the other hand being sensitive to new physics contributions they could give the first signals of new physics. The fact that $\left|V_{t s}\right|$ is already reasonably well known will be helpful in this context.

\subsection{Phase 2 (2007-2009)}

With the B-factories and Tevatron entering their mature stage and LHCB, $\mathrm{BTeV}$, Atlas and CMS beginning hopefully their operation, the quantities in (190) should offer a very good determination of the CKM matrix. I expect that other decays listed in Phase 1 will become more useful in view of improved data and new theoretical ideas. The most important new developments to be expected in this phase will be clean measurements of the angle $\gamma$ at LHCB and BTeV in decays $B_{s} \rightarrow D_{s}^{+} K^{-}$and $\bar{B}_{s} \rightarrow D_{s}^{-} K^{+}$and an improved measurement of $\operatorname{Br}\left(K^{+} \rightarrow \pi^{+} \nu \bar{\nu}\right)$ by the CKM collaboration at Fermilab. Also other strategies discussed in Section 5.4 and possible measurements of rare B-decays sensitive to both $\left|V_{t s}\right|$ and $\left|V_{t d}\right|$ should play an important role. This phase should provide (in case the phase 1 did not do 
it) definite answer whether MFV is sufficient to describe the data or whether new flavour violating interactions are required.

At the end of this phase we should also have much more improved knowledge about $K_{\mathrm{L}} \rightarrow \pi^{0} \nu \bar{\nu}$ from KOPIO at Brookhaven and JHF in Japan.

However, the most interesting scenario would be the discovery of supersymmetry at LHC which could considerably reduce the uncertainty in the supersymmetric parameters necessary for the study of FCNC processes.

\subsection{Phase 3 (2009-2013)}

Here precise measurements of $\operatorname{Br}\left(K_{\mathrm{L}} \rightarrow \pi^{0} \nu \bar{\nu}\right)$ from KOPIO and JHF will be among the highlights. In addition the branching ratios for most of the decays studied in phases 1 and 2 will be known with much higher precision. This will allow not only a precision test of SM but also to identify the patterns of new physics contributions that I personnally expect should show up at this level of accuracy. The combination of these studies with the results from LHC that should signal some direct signs of new physics should allow a convincing identification of this new physics.

No doubt the next ten years should be very exciting but the real progress will require extreme joined efforts by theorists and experimentalists.

\section{Acknowledgements}

I would like to thank the organizers for inviting me to such a wonderful winter school and most enjoyable atmosphere. I would also like to thank Robert Fleischer, Matthias Jamin, Stefan Recksiegel and Achille Stocchi for discussions. The work presented here has been supported in part by the German Bundesministerium für Bildung und Forschung under the contract 05HT1WOA3 and the DFG Project Bu. 706/1-2.

\section{References}

1. N. Cabibbo, Phys. Rev. Lett. 10, 531 (1963)

2. M. Kobayashi and K. Maskawa, Prog. Theor. Phys. 49, 652 (1973)

3. M. Battaglia, A.J. Buras, P. Gambino, A. Stocchi et al., hep-ph/0304132

4. A.J. Buras, hep-ph/9806471 in Probing the Standard Model of Particle Interactions, eds. R. Gupta, A. Morel, E. de Rafael and F. David (Elsevier Science B.V., Amsterdam, 1998) pp. 281-539

5. A.J. Buras, hep-ph/9905437 in Electroweak Physics, eds. A. Astbury et al, (World Scientific 2000) pp. 1-83.

6. A.J. Buras, hep-ph/0101336 in proceedings of the International School of Subnuclear Physics, ed. A .Zichichi, (World Scientific 2001) pp. 200-337 2000.

7. A.J. Buras, AIP Conf. Proc. 623, 3 (2002)

8. G. Buchalla, A.J. Buras and M. Lautenbacher, Rev. Mod. Phys 68, 1125 (1996)

9. A.J. Buras and R. Fleischer, Adv. Ser. Direct. High. Energy Phys. 15, 65 (1998)

10. Heavy Flavours II, eds. A.J. Buras and M. Lindner, World Scientific, (1998) 
11. G. Branco, L. Lavoura and J. Silva, (1999), CP Violation, Oxford Science Publications, Clarendon Press, Oxford

12. I.I. Bigi and A.I. Sanda, (2000), CP Violation, Cambridge Monographs on Particle Physics, Nuclear Physics and Cosmology, Cambridge University Press, Cambridge.

13. The BaBar Physics Book, eds. P. Harrison and H. Quinn, (1998), SLAC report 504.

14. B Decays at the LHC, eds. P. Ball, R. Fleischer, G.F. Tartarelli, P. Vikas and G. Wilkinson, hep-ph/0003238

15. B Physics at the Tevatron, Run II and Beyond, K. Anikeev et al., hep-ph/0201071

16. R. Fleischer, Phys. Rep. 370, 537 (2002) Y. Nir, hep-ph/9911321 hep-ph/0109090 G. Buchalla, hep-ph/020292, hep-ph/0302145

17. H. Fritzsch and Z.Z. Xing, Prog. Part. Nucl. Phys. 45, 1 (2000)

18. L.L. Chau and W.-Y. Keung, Phys. Rev. Lett. 53, 1802 (1984)

19. K. Hagiwara et al., "Review of Particle Physics", Phys. Rev. D 66, 010001 (2002)

20. L. Wolfenstein, Phys. Rev. Lett. 51, 1945 (1983)

21. A.J. Buras, M.E. Lautenbacher and G. Ostermaier, Phys. Rev. D 50, 3433 (1994)

22. M. Schmidtler and K.R. Schubert, Z. Phys. C 53, 347 (1992)

23. R. Aleksan, B. Kayser and D. London, Phys. Rev. Lett. 73, 18 (1994)

24. A.J. Buras, P. Gambino, M. Gorbahn, S. Jäger and L. Silvestrini, Phys. Lett. B500, 161 (2001)

25. C. Bobeth, T. Ewerth, F. Krüger and J. Urban, Phys. Rev. D66, 074021 (2002)

26. G. D'Ambrosio, et al., Nucl. Phys. B645, 155 (2002)

27. T. Inami and C.S. Lim, Progr. Theor. Phys. 65, 297 (1981)

28. A.J. Buras, hep-ph/0109197 in proceedings of Kaon 2001, eds. F. Costantini, G. Isodori, M. Sozzi, pp. 15-43

29. S.L. Glashow, J. Iliopoulos and L. Maiani Phys. Rev. D2, 1285 (1970)

30. J. Bijnens, J.-M. Gérard and G. Klein, Phys. Lett. B257, 191 (1991)

31. S. Herrlich and U. Nierste, Nucl. Phys. B419, 292 (1994)

32. L.L. Chau, Physics Reports, 95, 1 (1983)

33. J. Gasser and U.G. Meissner, Phys. Lett. B258, 219 (1991)

34. A.J. Buras, M. Jamin, and P.H. Weisz, Nucl. Phys. B347, 491 (1990)

35. S. Herrlich and U. Nierste, Phys. Rev. D52, 6505 (1995) Nucl. Phys. B476, $27(1996)$

36. M. Jamin and U. Nierste, recent update.

37. J. Urban, F. Krauss, U. Jentschura and G. Soff, Nucl. Phys. B523, 40 (1998)

38. The numerical value on the r.h.s of (100) being proportional to $\lambda^{-10}$ is very sensitive to $\lambda$ but as $A \propto \lambda^{-2}$ and $P_{c}(\varepsilon) \propto \lambda^{-4}$ the constraint (100) depends only quadratically on $\lambda$.

39. B. Aubert et al., Phys. Rev. Lett. 89, 201802 (2002)

40. K. Abe et al., Phys. Rev. D66, 071102 (2002)

41. Y. Nir, Nucl. Phys. Proc. Suppl. 117, 111 (2003)

42. M. Ciuchini et al., JHEP 0107, 013 (2001)

43. A. Höcker, et al., Eur. Phys. J. C21, 225 (2001)

44. A.J. Buras, F. Parodi and A. Stocchi, JHEP 0301, 029 (2003)

45. A. Lai et al., Eur. Phys. J. C22, 231 (2001) J.R. Batley et al., Phys. Lett. B544, 97 (2002) 
46. A. Alavi-Harati et al., Phys. Rev. Lett. 83, 22 (1999) Phys. Rev. D67, 012005 (2003)

47. H. Burkhardt et al., Phys. Lett. B206, 169 (1988) G.D. Barr et al., Phys. Lett. B317, 233 (1993)

48. L.K. Gibbons et al., Phys. Rev. Lett. 70, 1203 (1993)

49. S. Bosch et al., Nucl. Phys. B 565, 3 (2000)

50. A.J. Buras et al., Nucl. Phys. B592, 55 (2001)

51. M. Ciuchini and G. Martinelli, Nucl. Phys. Proc. Suppl. 99B, 27 (2001)

52. T. Hambye et al., Nucl. Phys. B564, 391 (2000)

53. S. Bertolini, J.O. Eeg and M. Fabbrichesi, Phys. Rev. D63, 056009 (2001)

54. E. Pallante and A. Pich, Phys. Rev. Lett. 84, 2568 (2000) Nucl. Phys. B592, 294 (2001) E. Pallante, A. Pich and I. Scimemi, Nucl. Phys. B617, 441 (2001)

55. J. Bijnens, E. Gamiz and J. Prades, JHEP 10, 009 (2001)

56. M. Knecht, S. Peris and E. de Rafael, Phys. Lett. B508, 117 (2001) T. Hambye, S. Peris and E. de Rafael, hep-ph/0305104

57. S. Bertolini, M. Fabbrichesi and J.O. Eeg, Rev. Mod. Phys. 72, 65 (2000)

58. S. Bertolini, hep-ph/0206095

59. A.J. Buras, M. Jamin, M.E. Lautenbacher and P.H. Weisz, Nucl. Phys. B370, 69 (1992) Nucl. Phys. B400, 37 (1993) A.J. Buras, M. Jamin and M.E. Lautenbacher, Nucl. Phys. B400, 75 (1993)

60. A.J. Buras, M. Jamin and M.E. Lautenbacher, Nucl. Phys. B408, 209 (1993)

61. M. Ciuchini, E. Franco, G. Martinelli and Reina, Phys. Lett. B301, 263 (1993) Nucl. Phys. B415, 403 (1994)

62. A.J. Buras and M. Jamin, hep-ph/0306217

63. W.A. Bardeen, A.J. Buras and J.-M. Gérard, Phys. Lett. B180, 133 (1986) Nucl. Phys. B293, 787 (1987) Phys. Lett. B192, 138 (1987) Phys. Lett. B211, $343(1988)$

64. G. Buchalla, A.J. Buras and M.K. Harlander, Nucl. Phys. B 349, 1 (1991)

65. V. Cirigliano, A. Pich, G. Ecker, and H. Neufeld, hep-ph/0307030

66. Y.-Y. Keum, U. Nierste and A.I. Sanda, Phys. Lett. B457, 157 (1999)

67. RBC Collaboration, T. Blum et al., hep-lat/0110075 CP-PACS Collaboration, S. Aoki et al., Nucl. Phys. Proc. Suppl. 106B, 332 (2002)

68. SPQCDR Collaboration, D. Becirevic et al., hep-lat/0209136

69. V. Cirigliano et al., Phys. Lett. B555, 71 (2003)

70. S. Narison, Nucl. Phys. B593, 3 (2001)

71. A.J. Buras and J.-M. Gérard, Phys. Lett. B517, 129 (2001)

72. J.F Donoghue, in proceedings of Kaon 2001, eds. F. Costantini, G. Isodori, M. Sozzi, pp 93-106

73. J. Hein, C. Davies, G.P. Lepage, Q. Mason and H. Trottier, hep-lat/0209077

74. R. Gupta, talk presented at CKM matrix and Unitarity Triangle, Durham, April 2003

75. Y. Grossman, G. Isidori and M.P. Worah, Phys. Rev. D58, 057504 (1998)

76. B. Aubert et al., hep-ex/020770

77. K. Abe et al., hep-ex/0207098

78. R. Fleischer and T. Mannel, Phys. Lett. B511, 240 (2001) G. Hiller, Phys. Rev. D66, 071502 (2002) A. Datta, Phys. Rev. D66, 071702 (2002) M. Ciuchini and L. Silvestrini, Phys. Rev. Lett. 89, 231802 (2002) M. Raidal, Phys. Rev. Lett. 89, 231803 (2002) Y. Grossman, Z. Ligeti, Y. Nir and H. Quinn, hep-ph/0303171 S. Khalil and E. Kou, hep-ph/0307024 
79. M. Gronau and D. London, Phys. Rev. Lett. 65, 3381 (1990)

80. R. Fleischer and J. Matias, Phys. Rev. D66, 054009 (2002) M. Gronau and J.L. Rosner, Phys. Rev. D65, 093012 (2002) D. Atwood and A. Soni, hep-ph/0206045 M. Ciuchini et al., Nucl. Phys. B 501, 271 (1997)

81. R. Fleischer, G. Isidori and J. Matias, JHEP 0305, 053 (2003)

82. G. Buchalla and A.J. Buras, Phys. Lett. B333, 221 (1994) Phys. Rev. D54, 6782 (1996)

83. M. Beneke, G. Buchalla, M. Neubert and C.T. Sachrajda, Nucl. Phys. B 606 , 245 (2001) M. Gronau and J.L. Rosner, Phys. Rev. D65, 013004 (2002) Z. Luo and J.L. Rosner, Phys. Rev. D54, 054027 (2002)

84. R.G. Sachs, EFI-85-22 (unpublished); I. Dunietz and R.G. Sachs, Phys. Rev. D37, 3186 (1988) [E: Phys. Rev. D39, 3515 (1988)] I. Dunietz, Phys. Lett. 427, 179 (1998) M. Diehl and G. Hiller, Phys. Lett. 517, 125 (2001)

85. J.P. Silva et al., Phys. Rev. D67, 036004 (2003)

86. R. Aleksan, I. Dunietz and B. Kayser, Z. Phys. C54, 653 (1992) R. Fleischer and I. Dunietz, Phys. Lett. B387, 361 (1996) A.F. Falk and A.A. Petrov, Phys. Rev. Lett. 85, 252 (2000) D. London, N. Sinha and R. Sinha, Phys. Rev. Lett. 85, $1807(2000)$

87. M. Gronau and D. Wyler, Phys. Lett. B265, 172 (1991)

88. M. Gronau and D. London, Phys. Lett. B253, 483 (1991) I. Dunietz, Phys. Lett. B270, 75 (1991)

89. D. Atwood, I. Dunietz and A. Soni, Phys. Rev. Lett. B78, 3257 (1997)

90. R. Fleischer, Nucl. Phys. B659, 321 (2003), Phys. Lett. B562, 234 (2003), hep-ph/0304027

91. R. Fleischer and D. Wyler, Phys. Rev. D62, 057503 (2000)

92. R. Fleischer, Eur. Phys. J. C10, 299 (1999) Phys. Rev. D60, 073008 (1999)

93. R. Fleischer, Phys. Lett. B459, 306 (1999)

94. M. Gronau and J.L. Rosner, Phys. Lett. B482, 71 (2000) C.W. Chiang and L. Wolfenstein, Phys. Lett. B493, 73 (2000)

95. M. Gronau, Phys. Lett. B492, 297 (2000)

96. R. Fleischer, Phys. Lett. B365, 399 (1996) R. Fleischer and T. Mannel, Phys. Rev., D57 2752 (1998)

97. M. Gronau and J.L. Rosner, Phys. Rev. D57, 6843 (1998)

98. R. Fleischer, Eur. Phys. J. C6, 451 (1999)

99. A.J. Buras and R. Fleischer, Eur. Phys. J. C11, 93 (1999) Eur. Phys. J. C16, $97(2000)$

100. M. Neubert, JHEP 9902, 014 (1998)

101. M. Neubert and J.L. Rosner, Phys. Lett. B441, 403 (1998) Phys. Rev. Lett. 81, 5076 (1998)

102. M. Ciuchini et al., Nucl. Phys. B501, 271 (1997)

103. A.J. Buras and L. Silvestrini, Nucl. Phys. B569, 3 (2000)

104. M. Beneke, G. Buchalla, M. Neubert and C.T. Sachrajda, Phys. Rev. Lett. 83, 1914 (1999) Nucl. Phys. B 591, 313 (2000) hep-ph/0007256 Nucl. Phys., B606 245 (2001)

105. H. -Y. Cheng, H.-n. Li and K.-C. Yang Phys. Rev. D60, 094005 (1999) Y.-Y. Keum, H.-n. Li and A.I. Sanda, Phys. Lett. B504, 6 (2001) Phys. Rev. D63, 054008 (2001)

106. Ch.W. Bauer et al., Phys. Rev. D65, 054022 (2002) Phys. Rev. D66, 014017 (2002) Phys. Rev. D66, 054005 (2002) I.W. Stewart, hep-ph/0208034 hep-ph/0109045 M. Beneke et al., Nucl. Phys. B 643, 431 (2002) Phys. Lett. B553, 267 (2003) 
107. A. Khodjamirian, Nucl. Phys. B 605, 558 (2001) hep-ph/0108205R. Rückl, S. Weinzierl and O. Yakovlev, hep-ph/0105161 hep-ph/0007344 A. Khodjamirian, T. Mannel and P. Urban, Phys. Rev. D67, 054027 (2003) A. Khodjamirian, T. Mannel and B. Melic, hep-ph/0304179

108. M. Gronau, J.l. Rosner and D. London, Phys. Rev. Lett. 73, 21 (1994)

109. D. Cronin-Hennessy et al., (CLEO), Phys. Rev. Lett. 85, 515 and 525 (2000) X.-G. He, W.-S. Hou and K-Ch. Yang, hep-ph/9902256 W.-S. Hou and K.Ch. Yang, Phys. Rev. D61, 073014 (2000) W.-S. Hou, J.G. Smith and F. Würthwein, hep-ex/9910014

110. M. Neubert, hep-ph/0207327

111. M. Ciuchini et al., Phys. Lett. 515, 33 (2001)

112. D. Rein and L.M. Sehgal, Phys. Rev. D39, 3325 (1989) J.S. Hagelin and L.S. Littenberg, Prog. Part. Nucl. Phys. 23, 1 (1989) M. Lu and M.B. Wise, Phys. Lett. B324, 461 (1994) S. Fajfer, hep-ph/9602322; C.Q. Geng, I.J. Hsu and Y.C. Lin, Phys. Rev. D54, 877 (1996) G. Buchalla and G. Isidori, Phys. Lett. B440, 170 (1998) A.F. Falk, A. Lewandowski and A.A. Petrov, Phys. Lett. B505, 107 (2001)

113. G. Buchalla and A.J. Buras, Nucl. Phys. B 400, 225 (1993) Nucl. Phys. B 412, 106 (1994)

114. G. Buchalla and A.J. Buras, Nucl. Phys. B 548, 309 (1999)

115. M. Misiak and J. Urban, Phys. Lett. B541, 161 (1999)

116. L. Littenberg, Phys. Rev. D39, 3322 (1989)

117. W. Marciano and Z. Parsa, Phys. Rev. D53, R1 (1996).

118. G. Isidori, hep-ph/0307014

119. S. Kettell, L. Landsberg and H. Nguyen, hep-ph/0212321

120. S. Adler et al., Phys. Rev. Lett. 79, 2204 (1997) Phys. Rev. Lett. 84, 3768 (2000) Phys. Rev. Lett. 88, 041803 (2002)

121. G. D'Ambrosio and G. Isidori, Phys. Lett. B530, 108 (2002) hep-ph/0112135

122. A. Alavi-Harati et al., Phys. Rev. D61, 072006 (2000)

123. Y. Grossman and Y. Nir, Phys. Lett. B398, 163 (1997)

124. L. Littenberg, hep-ex/0010048 T.K. Komatsubara, hep-ex/0112016

125. A. Belyaev et al, hep-ph/0107046

126. A.J. Buras and R. Buras, Phys. Lett. B501, 223 (2001) S. Bergmann and G. Perez, Phys. Rev. D64, 115009 (2001) JHEP 0008, 034 (2000) S. Laplace, Z. Ligeti, Y. Nir and G. Perez, Phys. Rev. D65, 094040 (2002)

127. A.J. Buras and R. Fleischer, Phys. Rev. D64, 115010 (2001)

128. A.J. Buras, hep-ph/303060.

129. A. Ali et al., Phys. Rev. D 66, 034002 (2002)

130. T. Appelquist et al., Phys. Rev. D 64, 035002 (2001)

131. A.J. Buras, M. Spranger and A. Weiler, Nucl. Phys. B 660, 225 (2003)

132. A.J. Buras, A. Poschenrieder, M. Spranger and A. Weiler, hep-ph/0306158

133. A.J. Buras, A. Poschenrieder, M. Spranger and A. Weiler, hep-ph/0307202 